\title{
The Stochastic SI Model with Recruitment and Deaths I. Comparison with the Closed SIS Model
}

\author{
JOHN A. JACQUEZ \\ Departments of Physiology and Biostatistics \\ AND \\ CARL P. SIMON \\ Departments of Mathematics, Economics, and Public Policy, \\ The University of Michigan, Ann Arbor, Michigan
}

Received 14 July 1992; revised 23 December 1992

\begin{abstract}
We compare the stochastic and deterministic versions of an SI model with recruitment, background deaths, and deaths due to the disease. For the stochastic version, analysis of the mean number of susceptibles, $m_{\mathscr{X}}$, and infecteds, $m_{\mathscr{Y}}$, and of the means conditioned on nonextinction of the infection, $m_{\mathscr{X}}^{*}$ and $m_{\mathscr{Y}}^{*}$, shows that (1) if $R_{0} \leqslant 1$, the disease dies out monotonically for the deterministic and stochastic models, and (2) if $R_{0}>1$, the disease dies out early with a probability close to $\left(1 / R_{0}\right)^{a}$, where $a$ is the number of infecteds introduced, or $m_{y}$ rises to a peak and then dies out slowly. For small populations $N$, the peak is an obvious maximum. If $N \geqslant 100$, the peak in $m_{y}$ is hidden in a long, nearly stationary plateau and $m_{y}^{*}$ is close to the deterministic endemic level for a large range of parameter values. The analytical results are illustrated with simulations. The results for the SI model are motivated by and compared with the corresponding results for the closed SIS model.
\end{abstract}

\section{INTRODUCTION}

In a previous paper in this series, Jacquez and O'Neill [6] examined the role of the basic reproduction number, also called basic reproduction ratio [4], $R_{0}$, in deterministic and stochastic SI models of homogeneous populations that have recruitment, deaths due to the disease, and background deaths. If $R_{0}<1$, the epidemic dies out, in the deterministic model, the number of infecteds decreases monotonically to zero as time progresses, and in the stochastic model the mean number of infecteds decreases monotonically to zero and, in fact, the probability of there being an infected in the population goes to zero. If $R_{0}>1$, the results differ slightly between the two models. In the deterministic 
model, the number of infecteds increases to a stationary level, the endemic level, which is globally asymptotically stable [21]. In the stochastic model, there is a nonzero probability that the epidemic will die out early in the process and a nonzero probability that the number of infecteds will increase to a long-lived quasi-stationary state the mean of which is close to the deterministic endemic level; these results are examined in more detail in this paper. A point not taken into account in [6] is that the long-term probability of extinction of the disease in the stochastic model is 1; however, for populations of size greater than 100 that extinction takes a very long time to occur.

The goal of this paper is to extend and correct the analytical and simulation results reported by Jacquez and O'Neill [6] so as to provide a detailed comparison of the behaviors of the deterministic and stochastic SI models with recruitment and with disease-related and non-diseaserelated deaths. We show how the results for each depend on the basic reproduction number, $R_{0}$. In brief, our simulations for the stochastic model for $R_{0}>1$ show the following.

(1) Early in the process, the probability of extinction of the epidemic, $P_{\text {ext }}$, quickly rises to a value that is near $\left(1 / R_{0}\right)^{a}$, where $a$ is the initial number of infectives introduced into the population. The reason this occurs in a nonlinear model is related to the next point.

(2) The probability mass distribution, $\mathscr{Y}$, for the number of infectives splits fairly early into a single mass at $\mathscr{Y}=0$, that is, extinction of the disease, well separated from a symmetric probability mass distribution for $\mathscr{Y}>0$.

(3) The quasi-stationary mean number of infectives conditioned on nonextinction is very close to the deterministic endemic value for a wide range of parameter values. The reason for that is shown analytically.

(4) The probability of extinction remains at a relatively flat level for a long period corresponding to the period during which the quasistationary solution is a good representation of the dynamics. But eventually $P_{\text {ext }}$ drifts up toward 1 . Simulations show that drift for small initial population sizes, less than about 50 . The larger the population, the longer it takes before that drift is perceptible; and for initial populations greater than 100 , that may be very long indeed.

This paper is organized in the following manner. Section II gives background results on two basic stochastic epidemic models. Section III summarizes analytical results on the deterministic and stochastic versions of the SI model under study and compares them. In Section IV, we present the results of simulations based on solving the systems of ODEs for the state probabilities in the stochastic model. In the last section we integrate the analytical results, simulations, and background. 


\section{BACKGROUND FOR THE STOCHASTIC MODEL}

Most of the previous work on stochastic epidemic models has been on two models: the closed SIS model and the general stochastic epidemic.

\section{A. SIS Model with Constant Population}

We begin by reviewing the simplest SIS model with constant population. Our review has gained considerably from the presentations on the SIS model given by Nåsell [16] and Martin-Löf [15] at the Luminy workshop in November 1991 and from conversations with both at the workshop. They also introduced us to the paper by Kryscio and Lefèvre [11] that presents the current state of the art for this model.

\section{DETERMINISTIC SIS MODEL}

A compartmental diagram showing the transitions in the SIS model is shown in Figure 1. A constant population of size $N$ is partitioned into $X$ susceptibles and $Y$ infectives. The differential equations for disease transmission are

$$
\dot{X}=-\lambda X(Y / N)+\delta Y
$$

and

$$
\dot{Y}=+\lambda X(Y / N)-\delta Y,
$$

where $\lambda=c \beta, c$ is the average number of possibly disease-transmitting contacts per person per unit time, $\beta$ is the probability that any one such contact will transmit infection, and $\delta$ is the rate of recovery. As usual, $\dot{X}$ means $d X / d t$.

Since $1 / \delta$ is the average duration of the disease before recovery, the threshold $R_{0}=\lambda / \delta$ is the number of new infections caused by an infective over the course of the infectious period if all contacts are with

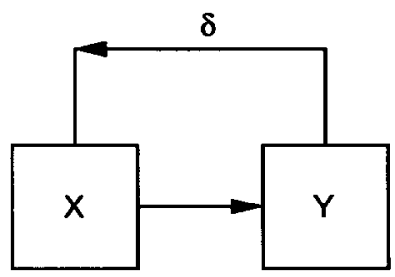

FIG. 1. Compartmental diagram for Equations (1) and (2). 
susceptibles. The parameter $R_{0}$ is the basic reproduction number for this model.

Equation (2) can be written as

$$
\dot{Y}=\delta\left[R_{0}(X / N)-1\right] Y .
$$

With the equation in this form, we can see that $R_{0}=1$ is a threshold that separates the case of the monotonic extinction of the disease from the case where the disease stabilizes at a positive (endemic) level. For, since $X / N \leqslant 1$ in (3), if $R_{0} \leqslant 1, \dot{Y}$ is always $\leqslant 0$ and $t \mapsto Y(t)$ decreases monotonically to the disease-free equilibrium at $Y=0$.

On the other hand, if $R_{0}>1$ and $X_{0}=X\left(t_{0}\right)$ is close enough to $N$ that $R_{0}\left(X_{0} / N\right)>1$, then $\dot{Y}\left(t_{0}\right)>0$, that is, the disease increases, at least in the beginning. Since the discase diminishes when $R_{0}(X / N)<1$, a stable endemic equilibrium occurs at $\left(X^{e}, Y^{e}\right)$, where

$$
X^{e} / N=1 / R_{0} .
$$

The result that, for $R_{0}>1$, the fraction of the population that is uninfected equals $1 / R_{0}$ at the endemic equilibrium is a key fact in all the models we will examine-both deterministic and stochastic. It follows that for the equilibrium infected fraction,

$$
Y^{e} / N=1-1 / R_{0} .
$$

If at the start of the process, there are $Y=a$ infectives, the disease will increase if $a / N<1-1 / R_{0}$ and will decrease if $a / N>1-1 / R_{0}$.

Let us look at these results in another way by converting system (1), (2) to a single equation. Since $\dot{N}=\dot{X}+\dot{Y}=0, N$ is constant over time, and we can replace $X$ by $N-Y$ in Equation (2) to obtain the following scalar logistic differential equation for $Y$ :

$$
\begin{aligned}
\dot{Y} & =\lambda(N-Y) \frac{Y}{N}-\delta Y=Y\left[(\lambda-\delta)-\frac{\lambda}{N} Y\right] \\
& =\lambda Y\left[\left(1-\frac{1}{R_{0}}\right)-\frac{Y}{N}\right] .
\end{aligned}
$$

Equation (6) has two equilibria: $Y_{1}^{e}=0$ and

$$
Y_{2}^{e}=\frac{(\lambda-\delta) N}{\lambda}=\left(1-\frac{1}{R_{0}}\right) N
$$




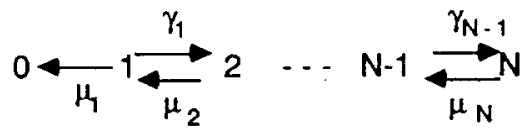

FIG. 2. Transition diagram for model given by Equations (8).

Once again we see that if $R_{0} \leqslant 1$, so that $Y_{2}^{e}<0$, then $\dot{Y}<0$ when $Y>0$, and $Y_{1}^{e}=0$ is a globally asymptotically stable steady state of (6) for $Y \geqslant 0$. If $R_{0}>1$, then $Y_{2}^{e}$ in (7) is positive and is a globally asymptotically stable steady state of (6) for $Y>0$.

\section{STOCHASTIC MODEL}

We turn now to the stochastic version of this model; a diagram showing all possible states and transitions is shown in Figure 2. We continue to use $N$ for the fixed size of the population. Now the number of infectives is a random variate, $\mathscr{Y}$, with realizations $y$, which are restricted to integer values between 0 and $N$. In Figure $2, \mu_{y}$ is the transition rate for $\mathscr{Y}$ decreasing from $y$ to $y-1$ and $\gamma_{y}$ is the transition rate for $\mathscr{Y}$ increasing from $y$ to $y+1$. More precisely,

$$
\begin{aligned}
& \operatorname{Pr}\{\mathscr{Y}(t+\Delta t)=y-1 \mid \mathscr{Y}(t)=y\}=\mu_{y} \Delta t+o(\Delta t), \\
& \operatorname{Pr}\{\mathscr{Y}(t+\Delta t)=y+1 \mid \mathscr{Y}(t)=y\}=\gamma_{y} \Delta t+o(\Delta t), \\
& \operatorname{Pr}\{\mathscr{Y}(t+\Delta t)=k \mid \mathscr{Y}(t)=y\}=o(\Delta t) \quad \text { if } k \neq y-1, y, y+1 .
\end{aligned}
$$

If we write (6) as

$$
\dot{Y}=\lambda Y(1-Y / N)-\delta Y,
$$

then it is natural to set

$$
\gamma_{y}=\lambda y(1-y / N) \quad \text { and } \quad \mu_{y}=\delta y
$$

in the stochastic analog of (6) for discrete populations. Note that (9) implies that $\gamma_{0}=\gamma_{N}=0$ and $\mu_{0}=0$.

As will be seen when we develop the equations for the SI model, the fraction in Equation (9) should be $y /(N-1)$, but all of the work in the literature on this system uses $y / N$, and we follow that convention here. 
3. KOLMOGOROV FORWARD EQUATIONS

Model (8) is a classical stochastic birth-and-death process with birth rates $\gamma_{y}$ and death rates $\mu_{y}$ and independence of births and deaths. As usual in such models, we let $p_{y}(t) \equiv \operatorname{Pr}\{\mathscr{Y}(t)=y\}$ for $y=0, \ldots, N$. Then,

$$
\begin{aligned}
p_{y}(t+\Delta t)= & \sum_{k=0}^{N} \operatorname{Pr}\{\mathscr{Y}(t+\Delta t)=y \mid \mathscr{Y}(t)=k\} \cdot \operatorname{Pr}\{\mathscr{Y}(t)=k\} \\
= & {\left[\gamma_{y-1} \Delta t+o(\Delta t)\right] \cdot p_{y-1}(t)+\left[\mu_{y+1} \Delta t+o(\Delta t)\right] \cdot p_{y+1}(t) } \\
& +\left[1-\gamma_{y} \Delta t-\mu_{y} \Delta t+o(\Delta t)\right] \cdot p_{y}(t)+o(\Delta t) .
\end{aligned}
$$

We write these equations as

$$
\begin{aligned}
& \frac{p_{y}(t+\Delta t)-p_{y}(t)}{\Delta t} \\
& \quad=\gamma_{y-1} p_{y-1}(t)+\mu_{y+1} p_{y+1}(t)-\left(\gamma_{y}+\mu_{y}\right) p_{y}(t)+\frac{o(\Delta t)}{\Delta t}
\end{aligned}
$$

and, letting $\Delta t \rightarrow 0$, as

$$
\dot{p}_{y}(t)=\gamma_{y-1} p_{y-1}(t)-\left(\gamma_{y}+\mu_{y}\right) p_{y}(t)+\mu_{y+1} p_{y+1}(t),
$$

for $y=0,1, \ldots, N$, where $\gamma_{-1}=\gamma_{0}=\gamma_{N}=0$ and $\mu_{0}=\mu_{N+1}=0$. Equations (10) are the Kolmogorov forward equations for the stochastic SIS model (8) under consideration. Written out in matrix form, these become

$$
\frac{d}{d t}\left(\begin{array}{c}
p_{0} \\
p_{1} \\
p_{2} \\
p_{3} \\
\vdots \\
p_{N-1} \\
p_{N}
\end{array}\right)=\left(\begin{array}{ccccc}
0 & \mu_{1} & 0 & \cdots & 0 \\
0 & -\left(\gamma_{1}+\mu_{1}\right) & \mu_{2} & \cdots & 0 \\
0 & \gamma_{1} & -\left(\gamma_{2}+\mu_{2}\right) & \cdots & 0 \\
0 & 0 & \gamma_{2} & \cdots & 0 \\
\vdots & \vdots & \vdots & \ddots & \vdots \\
0 & 0 & 0 & \cdots & \mu_{N} \\
0 & 0 & 0 & \cdots & -\mu_{N}
\end{array}\right)\left(\begin{array}{c}
p_{0} \\
p_{1} \\
p_{2} \\
p_{3} \\
\vdots \\
p_{N-1} \\
p_{N}
\end{array}\right)
$$

which we abbreviate as

$$
\dot{\mathbf{p}}=A \mathbf{p} .
$$


The matrix $A$ is a compartmental matrix (nonpositive diagonal entries, nonnegative off-diagonal entries, and nonpositive column sums), whose first column has only zeros and whose columns each sum to zero [7]. The latter property reflects the fact that, for any solution $\mathbf{p}(t)$ of (11), $\sum_{i=0}^{N} p_{i}(t)=1$ for all $t$. One eigenvalue of $A$ is $r_{0}=0$ with eigenvector $\mathbf{e}_{0}=(1,0,0, \ldots, 0)^{T}$. All the other eigenvalues $r_{1}, \ldots, r_{N}$ have negative real parts. In fact, Picard [19] shows that the $r_{i}$ 's are all distinct real numbers and therefore have a complete set of corresponding eigenvectors, which we write as $\mathbf{v}_{1}, \ldots, \mathbf{v}_{N}$. The general solution of system (11) is

$$
\mathbf{p}(t)=\mathbf{e}_{0}+e^{r_{1} t} \mathbf{v}_{1}+\cdots+e^{r_{N} t} \mathbf{v}_{N} .
$$

Since each $e^{r_{i} t} \rightarrow 0$ as $t \rightarrow \infty$, every solution $\mathbf{p}(t)$ of (11) tends to $\mathbf{e}_{0}$ as $t \rightarrow \infty$. In other words, the probability that the SIS epidemic will become extinct goes to 1 as $t \rightarrow \infty$. From another point of view, $y=0$ is the only absorbing state of the Markov process in Figure 2, and therefore the system will eventually be absorbed into it (with probability 1 ).

\section{QUASI-STATIONARY SOLUTIONS}

Notice the different behaviors of the deterministic (6) and stochastic (11) models. In the deterministic model, if $R_{0}>1$, the disease stabilizes at its carrying capacity (or endemic equilibrium) $Y_{2}^{e}$ in (7). In the stochastic model (11), for any positive choice of parameters in (11), the disease eventually is extinguished.

However, computer simulations of the stochastic model (11) for a population of size $N=100$, for example, yield behavior that is much closer to what we would expect from the deterministic model. We continue to write $\mathbf{p}(t)$ for the probability distribution (12) that is the solution of system (11). Let $m_{\mathscr{y}}(t)$ denote the mean of $\mathscr{Y}$ in the distribution $\mathbf{p}(t)$ :

$$
m_{\mathscr{Y}}(t) \equiv \sum_{y=0}^{N} p_{y}(t) \cdot y .
$$

As we will demonstrate in Section II.A.8, if $R_{0}=\lambda / \delta \leqslant N /(N-1)$, then $m_{y}(t)$ decreases monotonically to 0 as $t \rightarrow \infty$. This situation is pictured in Figure 3. On the other hand, if $R_{0}>N /(N-1)$, the computer solution of (11) seems to converge to a bimodal distribution with one local maximum at $Y_{1}^{e}=0$ and another local maximum near $Y_{2}^{e}$, the stable stationary solution of the deterministic model (6), as illustrated in Figure 4. As one expects from the exact form (12) of the solution $\mathbf{p}(t)$, this bimodal distribution is a temporary phenomenon; 


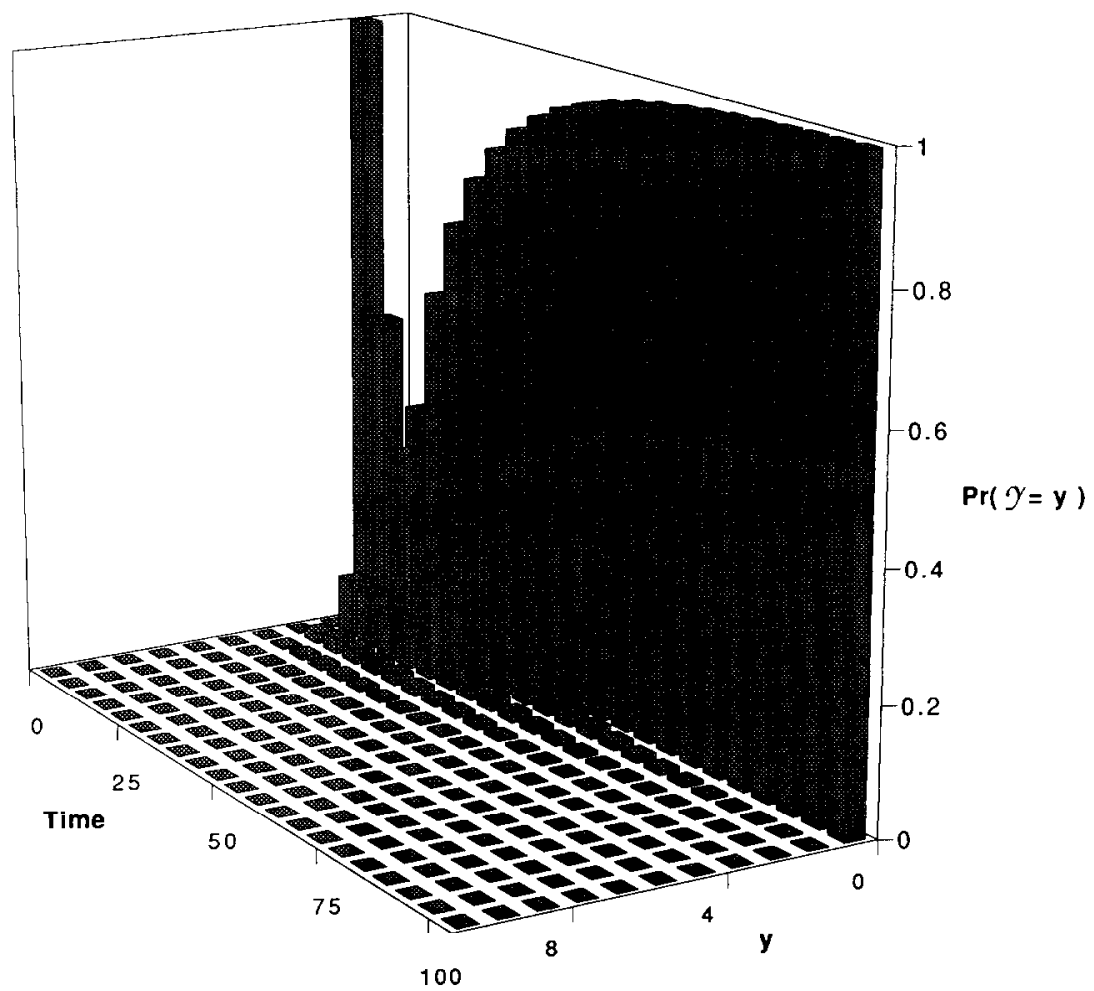

FIG. 3. Computer simulations of the solution distribution $\mathbf{p}(t)$ for $R_{0}=0.5$, $N=100, a=1$.

eventually the distribution $\mathbf{p}(t)$ converges to $\mathbf{e}_{0}$, and the disease dies out. But "eventually" can mean a very long time.

Since (11) is a continuous Markov chain with an absorbing state, one can compute the expected waiting time to absorption from any initial transient state. For $R_{0}>1$, Oppenheim et al. [18], Norden [17], and Kryscio and Lefèvre [11] derive the following lower and upper bounds for the expected time $\Gamma_{N, a}$ to absorption when the total population has size $N$ and $\mathscr{Y}(0)=a$, that is, $p_{a}(0)=1$. In these estimates, $\rho \equiv 1 / R_{0}=$ $\delta / \lambda$.

$$
\begin{aligned}
& \frac{\rho}{\delta(1-\rho)}\left(\frac{e^{-(1-\rho)}}{\rho}\right)^{N}\left(\frac{2 \pi}{N}\right)^{1 / 2} \\
& \quad \leqslant \Gamma_{N, a} \leqslant \frac{1}{\delta(1-\rho)}\left(\frac{e^{-(1-\rho)}}{\rho}\right)^{N}\left(\frac{2 \pi}{N}\right)^{1 / 2} \sum_{j=1}^{a} B_{N, j} \rho^{j}
\end{aligned}
$$




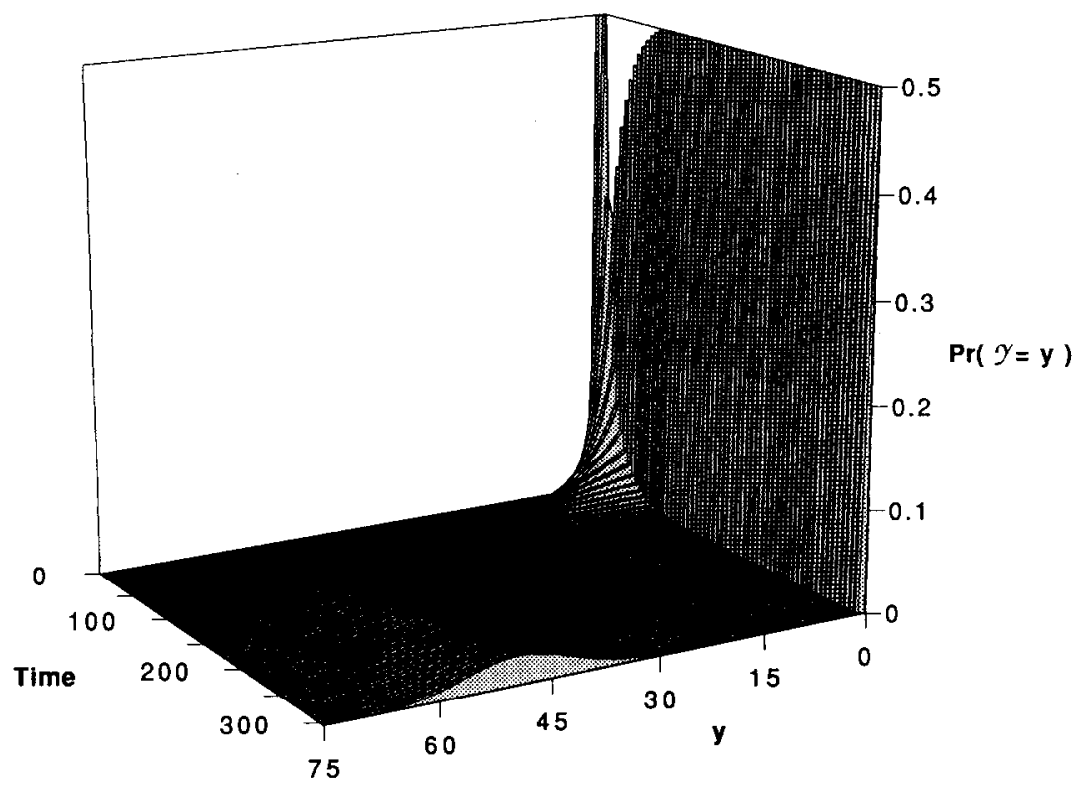

FIG. 4. Computer simulations of the solution distribution $\mathbf{p}(t)$ for $R_{0}=2.0$, $N=100, a=1$.

where $B_{N, j}=(N-j) ! N^{j} / N$ !. Note that $\Gamma_{N, a}$ goes to infinity exponentially in $N$ and that the lower bound in (14) is independent of $a$. See Weiss and Dishon [23] for a similar estimate for $a=N$. Kryscio and Lefèvre [11], who derive the upper bound in (14), argue that the upper bound is a better estimate of $\Gamma_{N, a}$ than the lower bound, especially for $N \geqslant 200$ and $R_{0} \geqslant 2$. For $N=200, \delta=0.1$, and $R_{0}=2, \mathrm{I}^{\prime}$ is of the order of $10^{17}$ [11].

In terms of the exact solution (12) to the Kolmogorov equations (11), order the eigenvalues $r_{i}$ of matrix $A$ so that

$$
r_{0}=0>r_{1}>r_{2}>\cdots .
$$

If $r_{1} \gg r_{2}$, so that $e^{-\left|r_{1}\right| t} \rightarrow 0$ much more slowly than $e^{-\left|r_{i}\right| t}$ for $i>1$, then

$$
\mathbf{p}(t) \equiv \mathbf{e}_{0}+e^{r_{1} t} \mathbf{v}_{1} \approx \mathbf{e}_{0}+\mathbf{v}_{1}
$$

will act like a "short-run" stationary solution for $r_{1} t$ small. This solution is called the quasi-stationary solution of system (12). If $r_{1}$ is very close to 0 , then $t$ can be fairly large and still have $r_{1} t$ rather small. 
5. CONDITIONING ON NONABSORPTION

When the expected time $\Gamma_{N, a}$ to absorption is large, it makes sense to study the quasi-stationary solution. In particular, we would like to find more effective ways to analyze it carefully without dealing with the approximation in (15).

One way to do this is to study the dynamics of the stochastic system (11) conditional on nonabsorption. We follow the vector $\left(p_{1}(t), \ldots, p_{N}(t)\right)$, normalized so that its components sum to 1 . To carry this out, define

$$
q_{i}(t)=\frac{p_{i}(t)}{1-p_{0}(t)} \quad \text { for } i=1, \ldots, N
$$

Then, writing $a_{i j}$ for the entries of the matrix $A$ in (11), we find

$$
\begin{aligned}
\dot{q}_{i} & =\frac{\dot{p}_{i}}{1-p_{0}}+\frac{p_{i}}{1-p_{0}} \cdot \frac{\dot{p}_{0}}{1-p_{0}} \\
& =\sum_{j=0}^{N} a_{i j} \frac{p_{j}}{1-p_{0}}+q_{i} \sum_{j=0}^{N} a_{0 j} \frac{p_{j}}{1-p_{0}} \\
& =\sum_{j=1}^{N} a_{i j} q_{j}+q_{i} \mu_{1} q_{1} .
\end{aligned}
$$

In other words, these $q_{i}(t)$ 's satisfy the system of quadratic differential equations

$$
\begin{aligned}
\left(\begin{array}{c}
\dot{q}_{1} \\
\dot{q}_{2} \\
\dot{q}_{3} \\
\vdots \\
\dot{q}_{N}
\end{array}\right)=\left(\begin{array}{cccc}
-\left(\gamma_{1}+\mu_{1}\right) & \mu_{2} & \ldots & 0 \\
\gamma_{1} & -\left(\gamma_{2}+\mu_{2}\right) & \cdots & 0 \\
0 & \gamma_{2} & \cdots & 0 \\
\vdots & \vdots & \ddots & \vdots \\
0 & 0 & \cdots & \mu_{N} \\
0 & 0 & \ldots & -\mu_{N}
\end{array}\right)\left(\begin{array}{c}
q_{1} \\
q_{2} \\
q_{3} \\
\vdots \\
q_{N}
\end{array}\right) \\
+\mu_{1} q_{1}\left(\begin{array}{c}
q_{1} \\
q_{2} \\
q_{3} \\
\vdots \\
q_{N}
\end{array}\right) .
\end{aligned}
$$

The steady state for this system is the solution $\mathbf{q}^{*}$ of the quadratic 
system of equations

$$
\begin{gathered}
\left(\begin{array}{ccccc}
-\left(\gamma_{1}+\mu_{1}\right) & \mu_{2} & 0 & \cdots & 0 \\
\gamma_{1} & -\left(\gamma_{2}+\mu_{2}\right) & \mu_{3} & \cdots & 0 \\
0 & \gamma_{2} & -\left(\gamma_{3}+\mu_{3}\right) & \cdots & 0 \\
0 & 0 & \gamma_{3} & \cdots & 0 \\
\vdots & \vdots & \vdots & \ddots & \vdots \\
0 & 0 & 0 & \cdots & -\mu_{N}
\end{array}\right)\left(\begin{array}{c}
q_{1}^{*} \\
q_{2}^{*} \\
q_{3}^{*} \\
\vdots \\
q_{N}^{*}
\end{array}\right) \\
=-\mu_{1} q_{1}^{*}\left(\begin{array}{c}
q_{1}^{*} \\
q_{2}^{*} \\
q_{3}^{*} \\
\vdots \\
q_{N}^{*}
\end{array}\right) .
\end{gathered}
$$

It follows that $\left(-1, q_{1}^{*}, q_{2}^{*}, \ldots, q_{N}^{*}\right)^{T}$ is the eigenvector $\mathbf{v}_{1}$ in (15) for the original matrix $A$ corresponding to the eigenvalue $r_{1}=-\mu_{1} q_{1}^{*}$. Put another way, if

$$
\left(\begin{array}{c}
q_{1}(t) \\
\vdots \\
q_{N}(t)
\end{array}\right)
$$

solves system (17), then

$$
\left(\begin{array}{c}
1 \\
0 \\
\vdots \\
0
\end{array}\right)+e^{-\mu_{1} q_{1}^{*} t}\left(\begin{array}{c}
-1 \\
q_{1}(t) \\
\vdots \\
q_{N}(t)
\end{array}\right)
$$

solves the original system (11). Therefore, the stationary solution $\mathbf{q}^{*}$ that satisfies (18) truly does correspond to the quasi-stationary solution $\left(0, \mathbf{q}^{*}\right)$ of (11).

In general, the stochastic system (11) will eventually hover around its quasi-stationary solution $\mathbf{q}^{*}$. By (19), it is easy to see that the expected waiting time to absorption starting from $\mathbf{p}(0)=\mathbf{q}^{*}$ is $1 / \mu_{1} q_{1}^{*}$, the absolute value of the reciprocal of the first nonzero eigenvalue of $A$. 
6. PREVENTING ABSORPTION

Another approach to the study of the quasi-stationary solution of (11) is to alter the stochastic system in Figure 2 by deleting the absorbing state, that is, by setting $\mu_{1}=0$. That gives us the modified Kolmogorov equations

$$
\begin{aligned}
\frac{d}{d t}\left(\begin{array}{c}
Q_{1} \\
Q_{2} \\
Q_{3} \\
Q_{4} \\
\vdots \\
Q_{N}
\end{array}\right)= & \left(\begin{array}{ccccc}
-\gamma_{1} & \mu_{2} & \cdots & 0 & 0 \\
\gamma_{1} & -\left(\gamma_{2}+\mu_{2}\right) & \cdots & 0 & 0 \\
0 & \gamma_{2} & \cdots & 0 & 0 \\
0 & 0 & \cdots & 0 & 0 \\
\vdots & \vdots & \ddots & \vdots & \vdots \\
0 & 0 & \cdots & -\left(\gamma_{N-1}+\mu_{N-1}\right) & \mu_{N} \\
0 & 0 & \cdots & \gamma_{N-1} & -\mu_{N}
\end{array}\right) \\
& \cdot\left(\begin{array}{c}
Q_{1} \\
Q_{2} \\
Q_{3} \\
Q_{4} \\
\vdots \\
Q_{N}
\end{array}\right) .
\end{aligned}
$$

This modified system is much easier to study than (11) or (17). For one thing, since system (20) is a regular Markov system, it has a unique steady-state distribution $\mathbf{Q}^{*}$. As Kryscio and Lefèvre [11] point out, one can set the right-hand side of system (20) equal to zero and solve recursively for the $Q_{n}^{*}$ 's in terms of $Q_{1}^{*}$ :

$$
Q_{n}^{*}=\frac{(N-1) !}{n(N-n) !}\left(\frac{1}{\rho N}\right)^{n-1} Q_{1}^{*} \quad \text { for } n=2, \ldots, N,
$$

with $Q_{1}^{*}$ chosen so that $\sum_{k=1}^{N} Q_{k}^{*}=1$ :

$$
Q_{1}^{*}=\left\{\sum_{k=1}^{N} \frac{(N-1) !}{k(N-k) !}\left(\frac{1}{\rho N}\right)^{k-1}\right\}^{-1}
$$

Kryscio and Lefèvre also show that this solution $\mathbf{Q}^{*}$ satisfies $\mathbf{Q}^{*} \sim \mathbf{q}^{*}$ as $N \rightarrow \infty$, where $\mathbf{q}^{*}$ is the solution of system (18). Oppenheim et al. [18] 
show that $\mathbf{Q}^{*}$ is sharply peaked at

$$
y=N(1-\rho)=N\left(1-1 / R_{0}\right)
$$

for $N$ large. Since $\mathbf{Q}^{*} \sim \mathbf{q}^{*}$, the same is true for the original absorbing process (11). Since the value of $y$ in (22) is precisely the value of the endemic equilibrium $Y_{2}^{e}$ in (7) for the deterministic equation (6), the stochastic process (11) does have a lot in common with the deterministic process (7).

\section{KURTZ'S APPROXIMATION THEOREM}

For large populations, one can get a handle on the quasi-stationary solutions of a stochastic population problem by using the theorems of Kurtz [12, 13], which tie together the deterministic and stochastic approaches. Roughly speaking, Kurtz uses the expectation of the transitions in the Markov process to approximate the stochastic dynamic by a deterministic one and then studies how well the trajectories of the deterministic dynamics approximate those of the stochastic process. More specifically, given a state $x$ of a Markov stochastic process, he constructs a deterministic dynamic by moving $X$ by the expected value of the set of all allowable transitions from $x$ in the Markov process. If the underlying model is a population model so that one actually keeps track of fractions of the total population, and if the lotal population $N$ is very large relative to the size of the expected transition $E(\Delta X)$ from any $x$, Kurtz's theorem, a variation of the law of large numbers, states that this deterministic dynamic is a good approximation of the stochastic process. For $N$ large enough, he then uses a form of the central limit theorem to compute the mean and standard deviation of this approximation.

THEOREM (Kurtz [12, Theorem 3.1])

Let $\mathbf{X}_{N}(t)$ be a one-parameter family of continuous-time Markov processes defined on the $m$-dimensional integer lattice $\mathbf{Z}^{\mathbf{m}}$. Suppose that there is a continuous function $f: \mathbf{R}^{\mathbf{m}} \times \mathbf{Z}^{\mathbf{m}} \rightarrow \mathbf{R}^{\mathbf{1}}$ that satisfies

$$
\operatorname{Pr}\left\{\mathbf{X}_{N}(t+\Delta t)=\mathbf{x}+\mathbf{k} \mid \mathbf{X}_{N}(t)=\mathbf{x}\right\}=N f\left(\frac{1}{N} \mathbf{x}, \mathbf{k}\right) \Delta t+o(\Delta t)
$$

for all positive integers $N$ and all positions $\mathbf{x}$ and increments $\mathbf{k}$ in $\mathbf{Z}^{\mathbf{m}}$. Define $F: \mathbf{R}^{\mathbf{m}} \rightarrow \mathbf{R}^{\mathbf{m}}$ by

$$
F(x) \equiv \sum_{\mathbf{k}} \mathbf{k} f(\mathbf{x}, \mathbf{k})=E(\Delta \mathbf{X} \mid \mathbf{X}=\mathbf{x})
$$


the expected change in $\mathbf{X}$ from $\mathbf{x}$. Suppose that there exists an open set $E$ in $\mathbf{R}^{\mathbf{m}}$ and a constant $M$ such that

(1) $|F(\mathbf{x})-F(\mathbf{y})| \leqslant M|\mathbf{x}-\mathbf{y}|$ for all $\mathbf{x}, \mathbf{y}$ in $\mathrm{E}$;

(2) $\sup _{\mathbf{x} \in E} \sum_{\mathbf{k}}|\mathbf{k}| f(\mathbf{x}, \mathbf{k})<\infty$;

(3) $\lim _{d \rightarrow \infty} \sup _{\mathbf{x} \in E} \sum_{|\mathbf{k}|>d}|\mathbf{k}| f(\mathbf{x}, \mathbf{k})=0$.

Let $\mathbf{Z}\left(t ; \mathbf{x}_{0}\right)$ be the solution of the (deterministic) initial value problem

$$
\frac{d \mathbf{Z}}{d t}=F(\mathbf{Z}), \quad \mathbf{Z}(0)=\mathbf{x}_{0}
$$

Suppose that $\mathbf{Z}\left(t ; \mathbf{x}_{0}\right) \in E$ for all $t \leqslant T$, and that $\lim _{N \rightarrow \infty}\left[\mathbf{X}_{N}(0) / N\right]=\mathbf{x}_{0}$ for the original family of Markov processes. Then, for every $\varepsilon>0$,

$$
\lim _{N \rightarrow \infty} \operatorname{Pr}\left\{\sup _{t \leqslant T}\left|\frac{1}{N} \mathbf{X}_{N}(t)-\mathbf{Z}\left(t ; \mathbf{x}_{0}\right)\right|>\varepsilon\right\}=0
$$

For the stochastic process (8), one checks easily that the rates $\gamma_{y}$ and $\mu_{y}$ in (9) satisfy the conditions of Kurtz's theorem. The expected deviation from $x$, Equation (24), is

$$
F(x)=(+1) \cdot \lambda x\left(1-\frac{x}{N}\right)+(-1) \cdot k x
$$

and the approximating differential equation (25) is the original logistic differential equation (6). Since all solutions of (6) tend to the deterministic "endemic equilibrium" $Y_{2}^{e}$ in (7), for $N$ large enough the "normalized" stochastic process converges weakly to a distribution sharply peaked at $Y_{2}^{e}$ by Kurtz's theorem. Martin-Löf [15] has used the Gaussian properties of the stochastic variate $Y(t)$ to estimate waiting time to absorption and has recovered estimates like those in (14).

8. ANALYSIS OF THE DYNAMICS OF $m_{\mathscr{y}}(t)$ AND $m_{y}^{*}(t)$

Kurtz's theorem provides a single deterministic differential equation that describes the dynamics of the stochastic system in Figure 2. However, it is useful only for $N$ large. To find a scalar deterministic dynamic for (8) that looks like (6) and holds for all $N$, we keep track of the mean $m_{y}(t)$ of $\mathscr{Y}$ relative to the solution $\mathbf{p}(t)$ of system (11):

$$
m_{\mathscr{Y}}(t)=\sum_{y=0}^{N} y \cdot p_{y}(t)
$$


One computes, using (11), that

$$
\frac{d m_{y}}{d t}=\sum_{y=0}^{N} y \frac{d p_{y}}{d t}=\lambda \sum_{y=0}^{N} y\left(1-\frac{y}{N}\right) p_{y}-\delta \sum_{y=0}^{N} y p_{y}
$$

An alternative approach to (28), and one that ties it more closely to the Kurtz approach, is to note that the expected value of the change in $\mathscr{y}$ conditional on the value $y$ is

$$
E[\mathscr{Y}(t+\Delta t)-\mathscr{Y}(t) \mid \mathscr{Y}(t)=y]=\lambda y\left(1-\frac{y}{N}\right) \Delta t-\delta y \Delta t
$$

Divide through (29) by $\Delta t$, and take the limit as $\Delta t \rightarrow 0$. Then take an expectation over the distribution of $\mathscr{Y}$ to obtain (28). Note that the right-hand side of (28) is the expected value of the additions to the number of infectives minus the expected value of the number of the deletions from the number of infectives.

At any critical point of $t \mapsto m_{y}(t)$-in particular, at any stationary point of (28) - the two terms on the right-hand side of (28) must equal each other. Write this condition as

$$
\frac{\lambda \sum_{y=0}^{N}(1-y / N) y p_{y}}{\delta \sum_{y=0}^{N} y p_{y}}=1 \quad \text { or } \quad \frac{\sum_{y=0}^{N}(1-y / N) y p_{y}}{\sum_{y=0}^{N} y p_{y}}=\frac{1}{R_{0}}
$$

Define $\xi(t)$ by

$$
\xi(t) \equiv \frac{\sum_{y=0}^{N}(1-y / N) y p_{y}}{\sum_{y=0}^{N} y p_{y}}=\frac{\sum_{y=1}^{N}(1-y / N) y p_{y}}{\sum_{y=1}^{N} y p_{y}}
$$

Since $1-y / N=x / N, \xi(t)$ can be considered an infective weighted average value of the uninfected fraction. Since $0 \leqslant(1-y / N) \leqslant 1$, then $0 \leqslant \xi(t) \leqslant 1$. Equation (28) can be rewritten as

$$
\frac{d m_{y}}{d t}=\delta\left[\frac{\lambda}{\delta} \cdot \frac{\sum_{y=0}^{N}(1-y / N) y p_{y}}{\sum_{y=0}^{N} y p_{y}} \cdot \sum_{y=0}^{N} y p_{y}-\sum_{y=0}^{N} y p_{y}\right] \text {, }
$$

which we write as

$$
\frac{d m_{y}}{d t}=\delta\left[R_{0} \xi(t)-1\right] m_{\mathscr{y}}
$$


or as

$$
\frac{d m_{Y}}{d t}=\lambda \sum_{y=1}^{N}\left[1-\frac{y}{N}-\frac{1}{R_{0}}\right] y p_{y} .
$$

Notice that (32) has the same form as Equation (3) for the deterministic model. The difference is that $\xi(t)$ replaces the deterministic $X / N$, but $\xi(t)$ is a weighted average of the terms $x / N$ in the stochastic model.

Thresholds. We look for thresholds by studying both initial growth rates and the stability of $m_{y}=0$ for ensembles of realizations. Since the numbers of infecteds and susceptibles can change only by integer steps, the threshold for the discrete stochastic model differs from the threshold for the deterministic model where the numbers of infecteds and susceptibles are continuous variables.

(a) Initial growth rate. If the initial condition is that $a$ infectives are present, then for $t=0, p_{a}(0)=1$, and $p_{y}(0)=0$ for $y \neq a$. Equation (28) then gives the relation

$$
\frac{d m_{Y}(0)}{d t}=\lambda\left[1-\frac{a}{N}-\frac{1}{R_{0}}\right] a .
$$

Thus, the initial growth is positive if $R_{0}>N /(N-a)$ and negative if $R_{0}<N /(N-a)$. Since the smallest value that $a$ can have is $a=1$ for the introduction of infection,

$$
R_{0}=N /(N-1)
$$

is the threshold for initial spread of the disease. Obviously, as $N \rightarrow \infty$, this threshold (35) for the discrete stochastic model approaches the threshold $R_{0}=1$ for the deterministic model.

(b) Mean for an ensemble of realizations. The largest coefficient in the summation on the right-hand side of Equation (33) is $1-1 / N-1 / R_{0}$. Hence, if that term is negative or zero, that is, when

$$
R_{0}<N /(N-1)
$$

then $d m_{\mathscr{y}} / d t<0$, and the ensemble average goes monotonically to zero. Stated another way, when $R_{0} \leqslant N /(N-1)$, the function

$$
M\left(p_{0}, p_{1}, \ldots, p_{N}\right)=\sum_{j=0}^{N} j p_{j}
$$


is a Lyapunov function for the global stability of $\mathbf{p}^{*}=(1,0, \ldots, 0)$ for system (11). See [21] for more details of this approach for deterministic epidemiological systems.

We now examine the places where $d m_{y} / d t$ is zero, in terms of the threshold (35).

(1) $R_{0}<N /(N-1)$. In this case, as we have just seen, the right-hand sides of Equations (32) and (33) are always negative, and $m_{y}(t)$ falls monotonically to the globally stable equilibrium at $m_{y}^{e}=0$.

(2) $R_{0}>N /(N-1)$. In this case, $d m_{\mathscr{y}} / d t$ can equal zero in two ways:

(a) The equilibrium $m_{y}^{e}=0$. The disease eventually dies out; but as already noted, if $N$ is large it may take a very long time for this to occur.

(b) The right-hand side of (32) is also zero when $R_{0} \xi-1=0$; let the value of $\xi$ at that point be $\xi^{m}$. (i) If $N$ is very small this is an obvious relative maximum of $t \mapsto m_{\mathscr{Y}}(t)$, where the expected number of additions to $\mathscr{Y}$ just balances the expected number of deletions from $\mathscr{Z}$. Written as $\xi^{m}=1 / R_{0}$, it says that at the peak of $t \mapsto m_{y}(t)$, the average uninfected fraction equals $1 / R_{0}$. This is the stochastic analog of (4). (ii) For larger $N$, this condition describes a not-so-obvious relative maximum in the long flat plateau of the graph of $m_{\mathscr{y}}$.

This discussion gives an interesting distinction between the model with a continuous population variable in Figure 1 and that with a discrete population variable in Figure 2. For the deterministic case, if $R_{0}>1$ and $Y(0)$ is close to $0, t \rightarrow Y(t)$ must increase initially before $Y$ converges to an endemic equilibrium. For the stochastic case, it is possible to have $R_{0}>1$ and yet have the mean value $m_{\mathscr{y}}$ decrease monotonically to 0 . This observation may play a role in studies of the behavior of the closed SIS model near $R_{0}=1$.

Mean Conditioned on Nonextinction. The mean conditioned on nonextinction of the infection can be written

$$
m_{y}^{*} \equiv \frac{\sum_{y=1}^{N} y p_{y}}{\sum_{y=1}^{N} p_{y}}=\sum_{y=1}^{N} y q_{y},
$$

where the $q_{y}$ 's are defined in (16). One can use (17) to compute that

$$
\begin{aligned}
\frac{d m_{y}^{*}}{d t} & =\sum_{y=1}^{N} \lambda y\left(1-\frac{y}{N}\right) q_{y}-\delta \sum_{y=1}^{N} y q_{y}+\delta q_{1} \sum_{y=1}^{N} y q_{y} \\
& =\left[\lambda \xi(t)-\delta+\delta q_{1}(t)\right] m_{y}^{*}=\delta\left[R_{0} \xi(t)-1+q_{1}(t)\right] m_{y}^{*} .
\end{aligned}
$$


At the equilibrium of $m_{\mathscr{y}}^{*}$, the mean of the quasi-stationary distribution of $\mathscr{Y}$, we have

$$
R_{0} \xi^{* e}-1=q_{1}^{e}
$$

where we write $\xi^{* e}$ for the value of $\xi$ at the quasi-stationary equilibrium. Since, as we have seen, $q_{1}^{e}$ goes to zero exponentially in $N$, we conclude that the average uninfected fraction at equilibrium $\xi^{* e}$ is approximately $1 / R_{0}$ at the quasi-stationary solution. Thus, $R_{0} \xi^{m}-1=$ 0 at the maximum in $m_{y}$ and $R_{0} \xi^{* e}-1=q_{1}^{e}$ at the quasi-stationary solution. However, $q_{1}^{e} \rightarrow 0$ as $N \rightarrow \infty$, and even for relatively small $N, N \geqslant 50, q_{1}^{e}$ is already very small. Thus $\xi^{* e} \rightarrow \xi^{m}=1 / R_{0}$ as $N$ increases.

In Figures 5-7 we show some simulation results to illustrate some of the properties of the SIS model.

Figure 5 shows the time course of the deterministic solution $Y$, the stochastic mean $m_{y}$, and the mean conditioned on nonextinction, $m_{y}^{*}$, for $R_{0}=0.5 . Y$ and $m_{y}$ fall monotonically to zero and are so close they are indistinguishable on the graph. Obviously one can calculate $m_{y}^{*}$ and obtain an apparent quasi-stationary solution, but it is of no value because the probability of extinction of the disease is steadily rising toward 1.

Figure 6 shows the time course of $Y, m_{y}$, and $m_{y}^{*}$ and of $\xi(t)$ and $R_{0} \xi(t)-1$ for $R_{0}=2$ and $N=10$. Notice that the mean conditioned on nonextinction differs some from the deterministic solution. Here $m_{y}$ peaks just before 50 time units and then falls steadily toward zero. Since there is no long near-stationary period, the quasi-stationary solution $m_{y}^{*}$ is irrelevant. Notice that $R_{0} \xi(t)-1$ is zero at just about the maximum

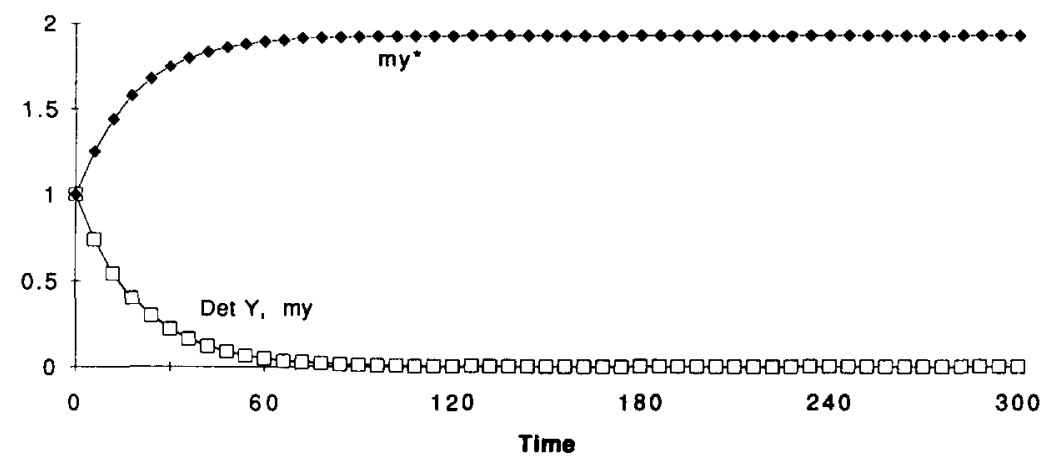

FIG. 5. Plots of $Y, m_{\mathscr{Y}}$, and $m_{\mathscr{Y}}^{*}$ for the closed SIS model with $R_{0}=0.5, N=100$, and $\boldsymbol{a}=1$. 

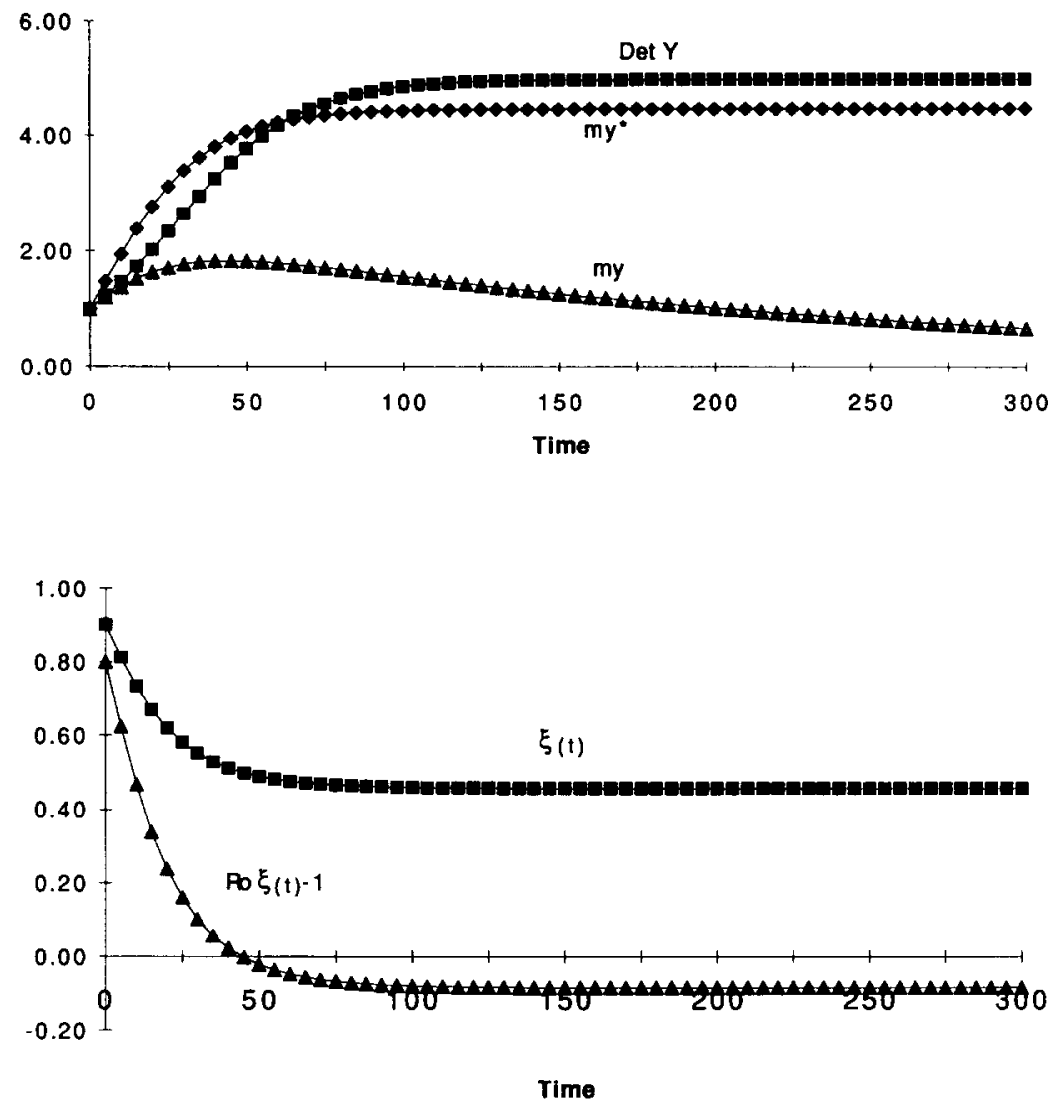

FIG. 6. Plots of $Y, m_{\mathscr{Y}}, m_{\mathscr{Y}}^{*}, \xi(t)$, and $R_{0} \xi(t)-1$ for the closed SIS model with $R_{0}=2, N=10$, and $a=1$.

in $m_{y}$. Figure 7 gives the same plot for $N=100$. Now, $m_{y}$ does have a long near-stationary period, and $m_{y}^{*}$ comes very close to $Y$. Although it is difficult to see on the graph, $R_{0} \xi(t)-1$ crosses zero at the peak in $m_{\mathscr{Y}}$.

B. The General Stochastic Epidemic

\section{THE DETERMINISTIC KERMACK-MCKENDRICK SYSTEM}

Another major focal point of the work on stochastic epidemic models has been the general stochastic model, a name given by Bailey [1] to the stochastic version of Kermack and McKendrick's [10] deterministic SIR model. Figure 8 presents the compartmental diagram for the Kermack- 

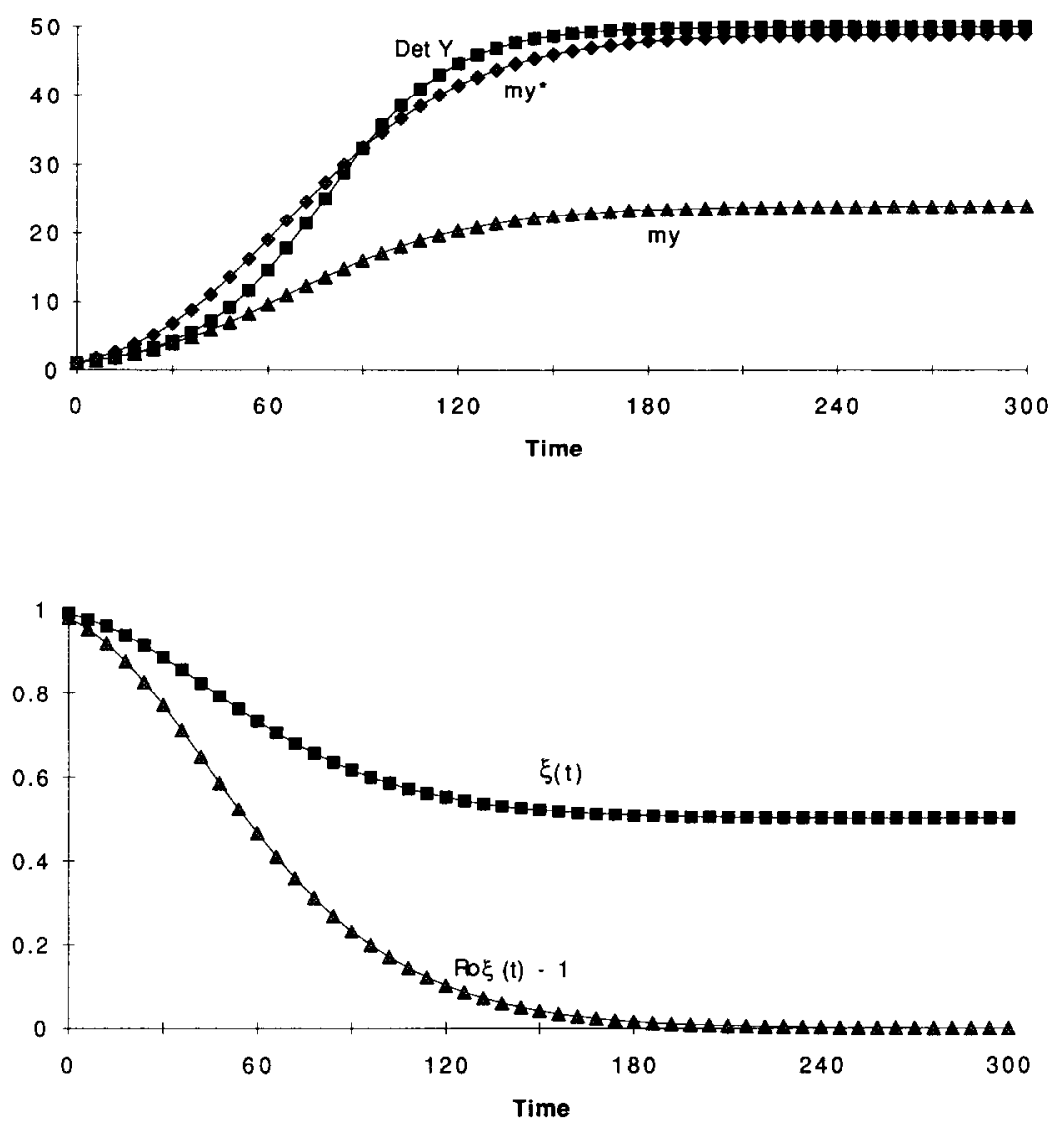

FIG. 7. Plots of $Y, m_{\mathscr{Y}}, m_{\mathscr{Y}}^{*}, \xi(t)$, and $R_{0} \xi(t)-1$ for the closed SIS model with $R_{0}=2, N=100$, and $a=1$.

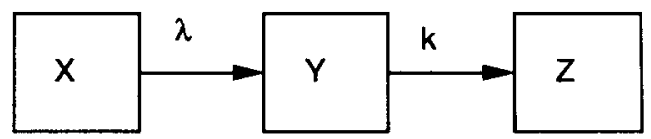

FIG. 8. The compartmental diagram for (37). 
McKendrick model. Its equations are

$$
\frac{d X}{d t}=-\lambda X \frac{Y}{N}, \quad \frac{d Y}{d t}=\lambda X \frac{Y}{N}-k Y, \quad \frac{d Z}{d t}=k Y,
$$

where $X, Y$, and $Z$ represent the numbers of susceptibles, infectives, and removeds, respectively. As before, let $N=X+Y+Z$ be the size of the population under study. Since $\dot{N}=0$ in (37), $N$ is constant for system (37), as it was for system (1), (2). As Jacquez and O'Neill [6] point out, $\lambda$ must vary as $1 / N$ in order to have a true SIR model in which the removeds play the role of the immunes and have an effect on the dynamics of the disease.

\section{GENERAL STOCHASTIC EPIDEMIC}

Once again, in forming the stochastic version of (37), we restrict the number of susceptibles and the number of infectives to be nonnegative integers. The conditional probabilities for the transitions are

$$
\begin{aligned}
& \operatorname{Pr}\{\mathscr{X}(t+\Delta t)=x-1, \mathscr{Y}(t+\Delta t)=y+1 \mid \mathscr{X}(t)=x, \mathscr{Y}(t)=y\} \\
&= \lambda x y \cdot \Delta t+o(\Delta t) \\
& \operatorname{Pr}\{\mathscr{X}(t+\Delta t)=x, \mathscr{Y}(t+\Delta t)=y-1 \mid \mathscr{X}(t)=x, \mathscr{Y}(t)=y\} \\
&= k y \cdot \Delta t+o(\Delta t) .
\end{aligned}
$$

As we did for the SIS model, we let $p_{x y}(t) \equiv \operatorname{Pr}\{\mathscr{Z}(t)=x, \mathscr{Y}(t)=y\}$. This time, we are led to the system of differential equations

$$
\begin{aligned}
\dot{p}_{x y}(t)= & \lambda(x+1)(y-1) p_{x+1, y-1}(t)-y(\lambda x+k) p_{x y}(t) \\
& +k(y+1) p_{x, y+1}(t)
\end{aligned}
$$

for nonnegative integers $x, y$ such that $x \leqslant n$ and $x+y \leqslant n+a$, where $n$ is the initial number of susceptibles and $a$ is the number of infectives introduced into the population; as before, $\mathscr{X}+\mathscr{Y}=N$. Jacquez and O'Neill [6] present a review of the general stochastic model (38); we do not repeat that here. We note, however, that the major results on thresholds, maximum size, final size, and duration of the epidemic depend on making $\lambda$ proportional to $1 / N$; then asymptotic results are obtained for large $N$ using, among other results, Kurtz's theorem.

Gani and Purdue [5] generalize the general stochastic epidemic by replacing the infection rate $\lambda x y$ by a generalized infection rate, $f_{x y}$, with the properties $f_{x 0}=f_{0 y}=0$. They then obtain the analog of Whittle's [24] threshold theorem for this model. Motivated by the transmission models for HIV, Ball and O'Neill [2] analyze a form of the general 
stochastic epidemic in which the transmission rate $\lambda x y$ is replaced by $\lambda x y /(x+y)$; they call that model the modified stochastic epidemic. They obtain threshold results corresponding to the results of Whittle [24] and Williams [25] for the general stochastic epidemic and also show that the modified epidemic spreads faster and infects more susceptibles. In this volume, Lefèvre and Picard [14] examine a model without recruitment in which infecteds either die or recover with immunity. The SIR general epidemic and the SI model without recruitment are the extremes for the risk of death 0 and 1 , respectively. In [9], we have compared the deterministic versions of SIS, SIR, SI, and SIRS models with recruitment, background deaths, and risk of death from the disease.

Bartlett [3] and later Stirzaker [22] introduced recruitment of susceptibles to the general stochastic model to examine oscillations in models of recurrent epidemics such as measles and chicken pox. Ridler-Rowe [20] looked at some of the properties of a general stochastic epidemic with recruitment into both susceptibles and infectives. So far, the only paper on properties of the SI model with the rate of infection given by $\lambda x y /(x+y)$, with recruitment of susceptibles, with background death, and with deaths due to the disease is that of Jacquez and O'Neill [6].

\section{THE SI MODEL: ANALYTIC RESULTS}

We turn now to the model on which this paper will focus, a model motivated by our interests in modeling the spread of HIV in homosexual communities. As we mentioned earlier, this is an SI model with

- Recruitment into the susceptible population,

- Death from the disease,

- Non-disease-related death, and

- Force of infection proportional to $X Y /(X+Y)$.

Aspects of HIV transmission missing from this model are progression through stages of the disease (with stage-dependent transmission probabilities) and multilevel population structure with an elaborate mixing matrix. See, for example, [8].

\section{A. The Deterministic Model}

\section{THE EQUATIONS OF THE MODEL}

Figure 9 gives the compartmental diagram of the states and transitions for the SI model of this study. As usual, $X$ and $Y$ are the numbers of susceptibles and infecteds, respectively, $U$ is the recruitment rate, $\mu$ is the rate constant for background deaths of susceptibles and infecteds, and $k$ is the rate constant for deaths due to the disease. 


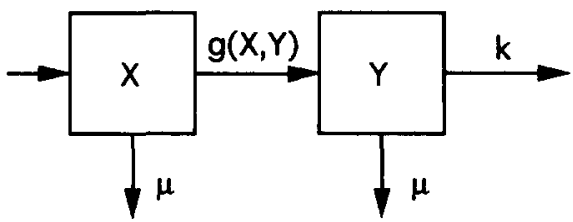

FIG. 9. The compartmental diagram for the SI system under study.

The force of infection is obtained as follows. Let $c$ be the number of possibly disease-transmitting contacts made per unit time per individual, and $\beta$ the probability of disease transmission for one such contact between an infected and a susceptible. Therefore, $\lambda=c \beta$ is the contact rate per individual, effective in transmission. The $X$ susceptibles make $\lambda X$ effective contacts per unit time. Under the assumption of random mixing, a fraction $Y /(X+Y)$ of these contacts are with infectives. Therefore, susceptibles are infected at the rate

$$
g(X, Y)=\lambda X Y /(X+Y) .
$$

The differential equations for $X$ and $Y$ are

$$
\begin{gathered}
\frac{d X}{d t}=-\lambda \frac{X Y}{X+Y}-\mu X+U, \\
\frac{d Y}{d t}=+\lambda \frac{X Y}{X+Y}-(k+\mu) Y
\end{gathered}
$$

In this model, $X$ and $Y$ are nonnegative continuous variables. As we noted for the simple SIS model in Section II, the basic reproduction number $R_{0}$ is defined by

$$
R_{0}=\frac{c \beta}{k+\mu}=\frac{\lambda}{k+\mu}
$$

the number of disease transmissions per infective during the course of his or her disease in a population of susceptibles. Equation (41) can now be written as

$$
\frac{d Y}{d t}=(k+\mu) Y\left(R_{0} \frac{X}{X+Y}-1\right)
$$


2. THE EQUILIBRIA OF THE MODEL AND THEIR STABILITY

As one can easily compute, system (40), (41) has two possible equilibria:

$$
\begin{gathered}
X^{o}=\frac{U}{\mu}, \quad Y^{o}=0, \\
X^{\mathrm{cq}}=\frac{U}{(k+\mu)\left(R_{0}-1\right)+\mu}=\frac{U}{\lambda-k}, \\
Y^{\mathrm{eq}}=\frac{\left(R_{0}-1\right) U}{(k+\mu)\left(R_{0}-1\right)+\mu}=\frac{U(\lambda-k-\mu)}{(k+\mu)(\lambda-k)} .
\end{gathered}
$$

If $R_{0} \leqslant 1$, then $\left(X^{o}, Y^{o}\right)$ in (44) is the only equilibrium of (40), (41), and every solution tends to it. For, in this case, as one can see readily by (43), $F(X, Y)=Y$ is a Lyapunov function for (40), (41), decreasing on all orbits. (See [9] and [21].) As initial conditions in our simulations of (40), (41), we use $X(0)=n$, where $n=U / \mu$ is the equilibrium state number of susceptibles for the system in the absence of infection and $Y(0)=a$, the number of infectives introduced into the population. In the simulation studies we worked with $a=1$ and $a=4$. As before, $N=X+Y$.

If $R_{0}>1$, system (40), (41) has both equilibria (44) and (45). The same function $F(X, Y)=Y$ is a Lyapunov function for the instability of $\left(X^{o}, Y^{\circ}\right)$. By setting the ratio of the two terms on the right-hand side of (41) equal to 1 , one computes easily that, as in all the models we have mentioned, at the endemic equilibrium the uninfected fraction is the reciprocal of $R_{0}$ :

$$
\frac{X^{\mathrm{eq}}}{X^{\mathrm{eq}}+Y^{\mathrm{eq}}}=\frac{1}{R_{0}}
$$

Figures 10 and 11 present the phase diagrams for the two cases $R_{0} \leqslant 1$ and $R_{0}>1$. As Figure 11 indicates, $\left(X^{\mathrm{eq}}, Y^{\mathrm{eq}}\right)$ is a globally stable equilibrium when $R_{0}>1$.

By adding Equations (40) and (41), onc computes that cvery cquilibrium $\left(X^{*}, Y^{*}\right)$ of this system falls on the line

$$
\mu X^{*}+(k+\mu) Y^{*}=U
$$

By (43) or (46), the endemic equilibrium (45) is at the intersection of this subspace and the ray

$$
R_{0} \frac{X}{X+\bar{Y}}=1
$$




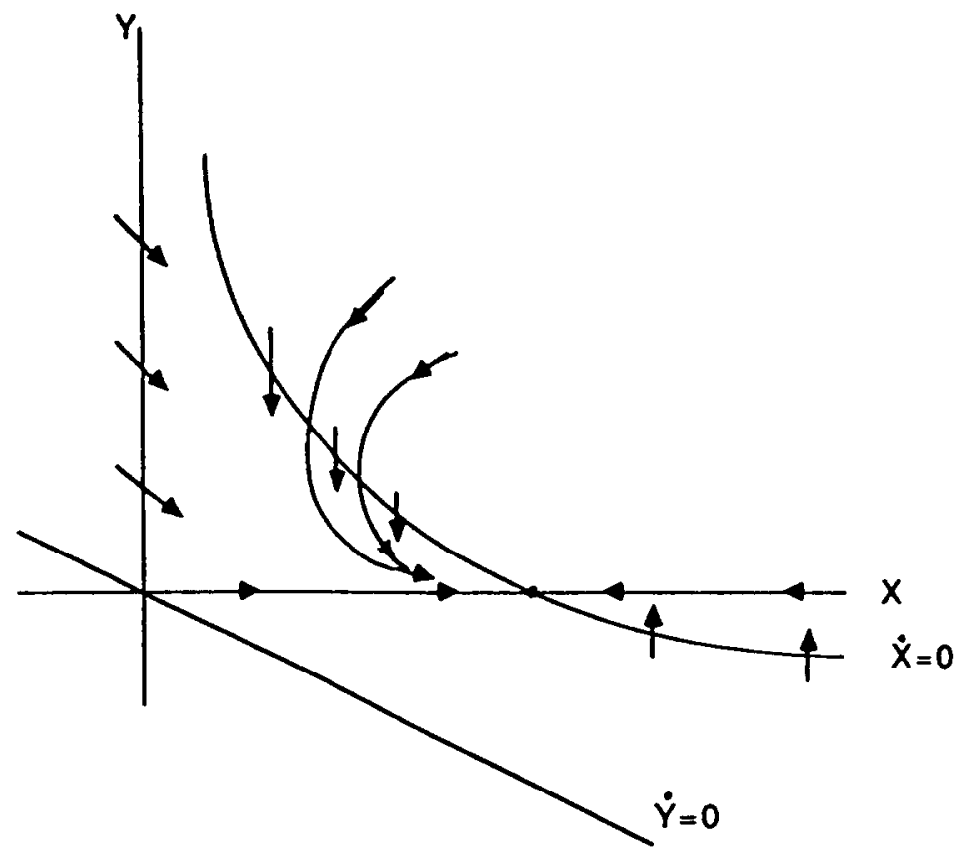

FIG. 10. Phase portrait for (40), (41) when $R_{0} \leqslant 1$.

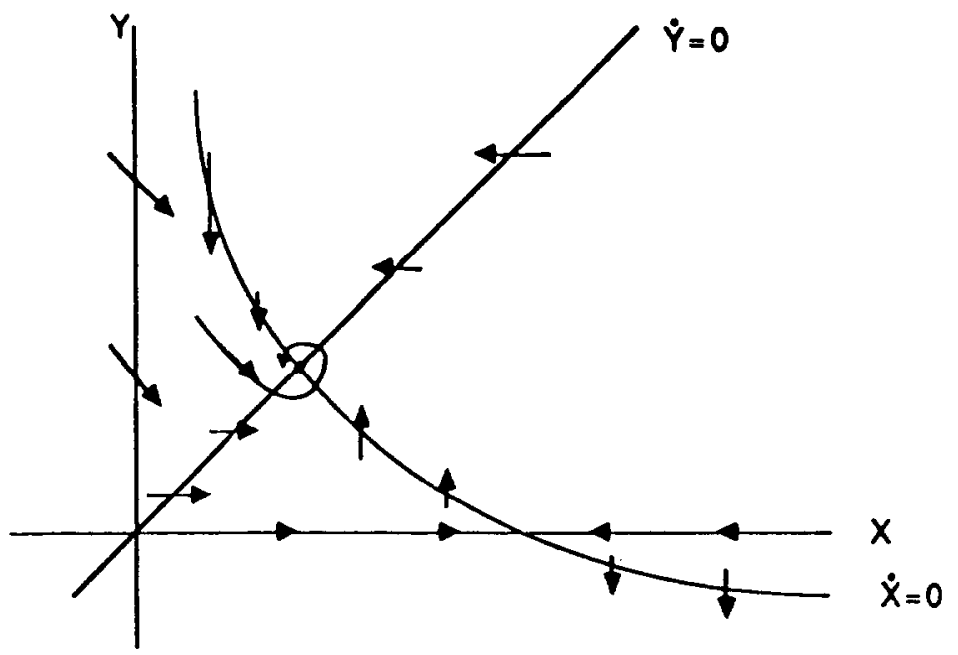

FIG. 11. Phase portrait for (40), (41) when $R_{0}>1$. 
B. The Stochastic Model

We turn now to the stochastic analog of the SI model diagramed in Figure 9. Again, for the stochastic model, $\mathscr{X}$ and $\mathscr{Y}$ are random variates with realizations $x$ and $y$ that are nonnegative integers. The transition rates from state $(x, y)$ are the following.

$\frac{x y}{x+y-1}$ : the rate at which a susceptible is converted to an infected. This transition rate must be assigned the value 0 whenever $y=0$ or $x=0$;

$U: \quad$ the rate for increase of $x$ by 1 by recruitment;

$\mu x$ : the rate of losing one susceptible to a competing cause of death;

$\mu y: \quad$ the rate of losing one infective to a competing cause of death;

ky: the rate of losing an infected due to the disease.

Figure 12 summarizes the transitions that can occur from any state $(x, y)$.

As we have done earlier, we write $p_{x y}(t)$ for the probability that the system is in state $(x, y)$ at time $t$. The corresponding Kolmogorov forward equations are

$$
\begin{aligned}
\frac{d p_{x y}}{d t}= & \lambda\left[\frac{(x+1)(y-1)}{x+y-1} p_{x+1, y-1}-\frac{x y}{x+y-1} p_{x y}\right]+U\left[p_{x-1, y}-p_{x y}\right] \\
& +(k+\mu)\left[(y+1) p_{x, y+1}-y p_{x y}\right]+\mu\left[(x+1) p_{x+1, y}-x p_{x y}\right] .
\end{aligned}
$$

(See Jacquez and O'Neill [6].) Since there cannot be a negative number of susceptibles or infectives, $p_{x y}$ must be zero if $x<0$ or $y<0$.

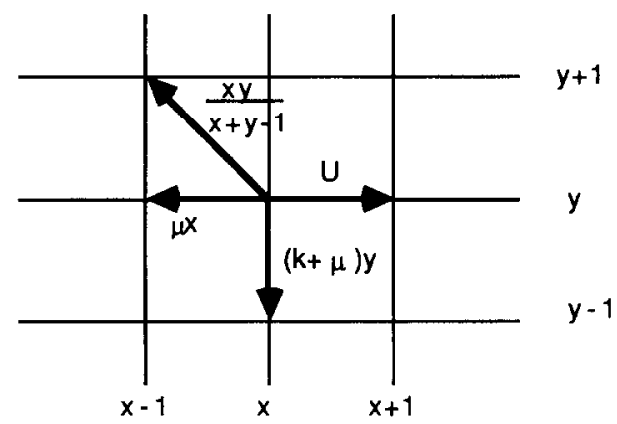

FIG. 12. The transitions that can occur at $(x, y)$. 
Remark. In the deterministic equations, $X$ and $Y$ are continuous variables, and the self-consistent formulation that leads to the differential equations (40), (41) has $X+Y$ in the denominator. To see this, set up the basic difference equations behind (40), (41); for example,

$$
\Delta X=-(c \Delta t) \beta \frac{X Y}{X+Y-\Delta X}-(\mu \Delta t) X+U \Delta t,
$$

divide through by $\Delta t$, and let $\Delta t \rightarrow 0$ to obtain (40). In the stochastic model, $\mathscr{Z}$ and $\mathscr{Y}$ can take on only integer values, so in state $(x, y)$ the probability that a susceptible's contact is with an infective must be $y /(x+y-1)$. In both cases, the susceptible must be taken out of the population before determining the fraction of those he might encounter that are infective. Jacquez and O'Neill [6] used $X+Y-1$ in the denominators of Equations (40) and (41) and so had to restrict the domain to $X+Y>1$. Although the difference is small for $N=X+Y$ large, we consider it more appropriate to compare the self-consistent formulations of the two models.

\section{MEANS}

As we did for the closed SIS model in Section II.A.8, we write a system of two deterministic differential equations that uses the mean number of susceptibles and infecteds to keep track of the stochastic dynamics (48):

$$
\begin{aligned}
& m_{\mathscr{X}}(t)=E[\mathscr{X}(t)]=\sum_{x, y=0}^{\infty} x p_{x y}(t), \\
& m_{\mathscr{Y}}(t)=E[\mathscr{Y}(t)]=\sum_{x, y=0}^{\infty} y p_{x y}(t) .
\end{aligned}
$$

The differential equations for $m_{\mathscr{X}}$ and $m_{\mathscr{Y}}$ are readily obtained from the differential equations (48) of the state probabilities [6], as we did in Equation (28) for the closed SIS model:

$$
\begin{aligned}
\frac{d m_{\mathscr{P}}}{d t} & =-\lambda \sum_{x=0}^{\infty} \sum_{y=0}^{\infty} \frac{x y}{x+y-1} p_{x y}-\mu \sum_{x=0}^{\infty} \sum_{y=0}^{\infty} x p_{x y}+U, \\
\frac{d m_{y}}{d t} & =\lambda \sum_{x=0}^{\infty} \sum_{y=0}^{\infty} \frac{x y}{x+y-1} p_{x y}-(k+\mu) \sum_{x=0}^{\infty} \sum_{y=0}^{\infty} y p_{x y} \\
& =(k+\mu) \sum_{x=0}^{\infty} \sum_{y=0}^{\infty}\left(R_{0} \frac{x}{x+y-1}-1\right) y p_{x y} .
\end{aligned}
$$


2. THRESHOLDS AND EQUILIBRIA FOR THE MEANS

For $R_{0} \leqslant 1$, we see from (53) that $d m_{\mathcal{Z}} / d t$ is always negative, and so $m_{y}(t)$ falls monotonically to zero as $t \rightarrow \infty$. Therefore, as Jacquez and O'Neill [6] point out, the stochastic equilibrium in this case is

$$
m_{\mathscr{X}}=\frac{U}{\mu}, \quad m_{\mathscr{Y}}=0,
$$

and it is globally asymptotically stable. At this equilibrium, $p_{x y}=0$ for all $y>0$; therefore, for $R_{0} \leqslant 1$, the deterministic and stochastic systems have the same equilibrium. In particular, as we saw for the closed SIS model, the function

$$
M(\mathbf{p})=\sum_{x, y} y p_{x y}
$$

is a Lyapunov function for the global stability of this equilibrium when $R_{0}<1$.

To study the dynamics for $R_{0}>1$, we first define two means that will be useful:

$$
\begin{gathered}
\xi(t)=\frac{\sum_{x=0} \sum_{y=0} \frac{x}{x+y-1} y p_{x y}}{\sum_{x=0} \sum_{y=0} y p_{x y}}=\frac{\sum_{x=0} \sum_{y=1} \frac{x}{x+y-1} y p_{x y}}{\sum_{x=0} \sum_{y=1} y p_{x y}} \\
\eta(t)=\frac{\sum_{x=0} \sum_{y=1} \frac{y}{x+y-1} x p_{x y}}{\sum_{x=0} \sum_{y=1} x p_{x y}} .
\end{gathered}
$$

Note that $\xi$ is a weighted average of the uninfected fraction $x /(x+y$ -1) with weights $y p_{x y}$ and $\eta$ is a weighted average of the infected fraction $y /(x+y-1)$ with weights $x p_{x y}$, so that $0 \leqslant \xi(t) \leqslant 1$ and $0 \leqslant$ $\eta(t) \leqslant 1$. Following Jacquez and O'Neill [6], we rewrite (51), (53) as

$$
\begin{aligned}
\frac{d m_{\mathscr{X}}}{d t} & =-\lambda \xi(t) m_{\mathscr{Y}}-\mu m_{\mathscr{X}}+U, \\
\frac{d m_{\mathscr{Y}}}{d t} & =(k+\mu)\left[R_{0} \xi(t)-1\right] m_{\mathscr{Y}} .
\end{aligned}
$$

We will use the notation $\xi^{m}$ and $\eta^{m}$ for the values of $\xi$ and $\eta$ at any zero of (57) where $m_{\mathscr{Y}}^{e} \neq 0$. Then, by (57),

$$
\xi^{m}=1 / R_{0},
$$

which can be viewed as the stochastic analog of (46). 
By setting the right-hand side of (56) equal to zero and substituting in (58), we note that any steady state $\left(m_{\mathscr{P}}^{e}, m_{\mathscr{Y}}^{e}\right)$ of $(56),(57)$ must satisfy

$$
U=\mu m_{\mathscr{Z}}^{e}+(k+\mu) m_{\mathscr{Y}}^{e},
$$

the stochastic analog of (47).

\section{STOCHASTIC DYNAMICS CONDITIONAL ON NONEXTINCTION}

Just as we did in our study of the closed SIS model in Section II.A, we keep track of the stochastic dynamics conditioned on nonextinction of the disease. These dynamics are important for two reasons. First, as will be seen for the simulations for $R_{0}>1$, the marginal distributions split fairly soon after the start of the process and become bimodal; the distribution of the number of infecteds then has an isolated mass at $y=0$ and, separated from that, an approximately symmetric peak with $y$ positive. Second, for $R_{0}>1$, some epidemics die out early and so should not be counted when one compares theoretical results with data on actual cpidemics.

For the stochastic system (48) under study, the states $\{(x, 0)\}_{x=0}^{\infty}$ form a communicating class (any one such state can be reached by any other in a finite number of steps), and this class is an absorbing class for the system.

We define the probability of extinction $P_{\text {ext }}$ of the disease, that is, the probability of absorption of the process, and the probability $P_{\text {inf }}$ that there is infection in the population:

$$
P_{\mathrm{ext}}(t)=\sum_{x=0} p_{x 0}(t) \quad \text { and } \quad P_{\mathrm{inf}}(t)=\sum_{x=0}^{\infty} \sum_{y=1}^{\infty} p_{x y}(t) .
$$

Of course, $P_{\mathrm{ext}}(t)+P_{\mathrm{inf}}(t)=1$.

We work out the dynamics of (48) conditioned on not reaching the absorbing class. For $x=0,1,2, \ldots$ and $y=1,2, \ldots$, let

$$
q_{x y} \equiv \frac{p_{x y}}{1-\sum_{i=0}^{\infty} p_{i 0}}=\frac{p_{x y}}{P_{\text {inf }}},
$$

the probability of being in state $(x, y)$, conditional on not having been absorbed. Let $a_{x y}^{i j}$ be the coefficient of $p_{i j}$ in the linear expression for $\dot{p}_{x y}$, Equation (48). Then, for $x \geqslant 0$ and $y \geqslant 1$,

$$
\begin{aligned}
\dot{q}_{x y} & =\frac{\dot{p}_{x y}}{1-\sum_{i=0}^{\infty} p_{i 0}}+\frac{p_{x y}}{1-\sum_{i=0}^{\infty} p_{i 0}}\left(\frac{\sum_{i=0}^{\infty} \dot{p}_{i 0}}{1-\sum_{i=0}^{\infty} p_{i 0}}\right) \\
& =\sum_{i=0}^{\infty} \sum_{j=1}^{\infty} a_{x y}^{i j} q_{i j}+q_{x y}(\mu+k) \sum_{i=0}^{\infty} q_{i 1} .
\end{aligned}
$$


This is the system that compares with (17) for the constant-population SIS model (11).

The means conditioned on nonextinction are

$$
m_{x p}^{*}(t)=\frac{\sum_{x=0}^{\infty} \sum_{y=1}^{\infty} x p_{x y}(t)}{\sum_{x=0}^{\infty} \sum_{y=1}^{\infty} p_{x y}(t)}=\sum_{x=0}^{\infty} \sum_{y=1}^{\infty} x q_{x y}
$$

and

$$
m_{Y}^{*}(t)=\frac{\sum_{x=0}^{\infty} \sum_{y=1}^{\infty} y p_{x y}(t)}{\sum_{x=0}^{\infty} \sum_{y=1}^{\infty} p_{x y}(t)}=\sum_{x=0}^{\infty} \sum_{y=1}^{\infty} y q_{x y} .
$$

One can use (61)-(63) to compute the rate of change of $m_{\mathscr{Z}}^{*}$ and $m_{\mathscr{Y}}^{*}$ with $t$ :

$$
\begin{gathered}
\frac{d m_{\mathscr{X}}^{*}}{d t}=U-\lambda \xi(t) m_{\mathscr{Y}}^{*}-\mu m_{\mathscr{X}}^{*}+(\mu+k)\left[P_{1}^{*} m_{\mathscr{X}}^{*}-\sum_{x=0}^{\infty} x q_{x 1}\right], \\
\frac{d m_{\mathscr{Y}}^{*}}{d t}=(\mu+k)\left[R_{0} \xi(t)-1+P_{1}^{*}(t)\right] m_{\mathscr{Y}}^{*},
\end{gathered}
$$

where

$$
P_{1}^{*}(t) \equiv \sum_{x=0}^{\infty} q_{x 1}=\operatorname{Pr}\{\mathscr{Y}=1 \mid \mathscr{Y} \neq 0\}
$$

the probability that $\mathscr{Y}=1$, conditional on nonextinction. Notice that the term in square brackets in (64) can also be written as $\Sigma_{x=0}\left(m_{\mathscr{X}}^{*}-x\right) q_{x 1}$.

Compare (64) and (65) with the corresponding Equations (56) and (57) for $d m_{\mathscr{X}} / d t$ and $d m_{\mathscr{Y}} / d t$. The only differences are in the $\sum q_{x 1}$ and $\sum x q_{x 1}$ terms. This is similar to the difference we found between Equations (32) and (36) for the closed SIS model, where the difference was the $q_{1}$ term. In particular, at the quasi-stationary solution, where $d m_{y}^{*} / d t=0$ and $m_{y}^{*} \neq 0$,

$$
\xi^{* e}=\frac{1}{R_{0}}+\frac{\sum_{x} q_{x 1}^{e}}{R_{0}}
$$

As occurred for the closed SIS model, we expect $\sum_{x} q_{x 1}^{e}$ to converge to 0 as $N \rightarrow \infty$, so that $\xi^{* e} \rightarrow \xi^{m}=1 / R_{0}$ as $N \rightarrow \infty$. Such convergence has been evident in all our simulations with $N \geqslant 100$.

Similarly, adding Equations (64) and (65), we note that at the 
quasi-stationary solution,

$$
U-\mu m_{\mathscr{X}}^{*}-(\mu+k) m_{\mathscr{Y}}^{*}=(\mu+k)\left[\sum_{x} x q_{x 1}-m_{N}^{*} \sum_{x} q_{x 1}\right],
$$

where $m_{N}^{*}=m_{\mathscr{X}}^{*}+m_{Y}^{*}$, the equilibrium mean population size conditioned on nonextinction. Once again, if the $\Sigma_{x} q_{x 1}$ and $\Sigma_{x} x q_{x 1}$ go to zero as $N \rightarrow \infty$, then for large $N,\left(m_{\mathscr{x}}^{*}, m_{\mathscr{Y}}^{*}\right)$ lies near the invariant subspace (47) that contains $\left(X^{\mathrm{eq}}, Y^{\mathrm{eq}}\right)$ and $\left(m_{\mathscr{P}}^{e}, m_{\mathscr{Y}}^{e}\right)$.

Rewrite (63) as $m_{y}^{*}=m_{y} / P_{\text {inf }}$. Then, differentiation yields

$$
\frac{d m_{y}^{*}}{d t}=\frac{1}{P_{\text {inf }}}\left[\frac{d m_{y}}{d t}+(k+\mu) \sum_{x=0}^{\infty} q_{x 1}\right] \text {. }
$$

Therefore, at any critical point of $m_{\mathscr{Y}}, m_{\mathscr{Y}}^{*}$ is increasing; at any critical point of $m_{\mathscr{Y}}^{*}, m_{\mathscr{Y}}$ is decreasing. In particular, $m_{y}$ will hit its maximal value before the system reaches its quasi-stationary solution. In addition, $d m_{y} / d t \rightarrow P_{\text {inf }} d m_{\mathscr{Y}}^{*} / d t$ as $N \rightarrow \infty$.

\section{QUASI-STATIONARY SOLUTIONS: KURTZ'S APPROACH}

In Section II.A.7, we used Kurtz's approximation theorem to approximate a normalized version of the stochastic dynamics (11) by a deterministic differential equation (25), (26). In this approximation, the quasi-stationary solution of (11) corresponds to the endemic equilibrium of (25). It is, of course, an asymptotic result for $N$ large.

In this section, we will use Kurtz's theorem to draw for system (48) the same conclusions that were drawn for system (11):

For $U$ large, the quasi-stationary solution of (48) is a distribution that is sharply peaked at $Y^{e}$ in (45), so the behavior of the stochastic dynamic (48) is very similar to the behavior of its deterministic counterpart (40), (41), both for $R_{0} \leqslant 1$ and for $R_{0}>1$.

In the notation of Kurtz's theorem, we will use $U=n \mu$, the recruitment rate, as the parameter $N$ in the statement of the theorem. This makes sense since we consider the models as starting at the disease-free equilibrium $\mathscr{Z}_{0}=n=U / \mu$. We set

$$
\begin{gathered}
f((x, y),(1,0))=1, \\
f((x, y),(-1,0))=\mu x, \quad f((x, y),(0,-1))=(\mu+k) y, \\
f((x, y),(-1,1))=\frac{\lambda x y}{x+(y-1)} .
\end{gathered}
$$


The last three expressions are homogeneous of degree +1 , so they clearly satisfy

$$
U \cdot f\left(\frac{1}{U}(x, y),\left(k_{1}, k_{2}\right)\right)=f\left((x, y),\left(k_{1}, k_{2}\right)\right) .
$$

Furthermore,

$$
U \cdot f\left(\frac{1}{U}(x, y),(1,0)\right)=U \cdot 1=U
$$

so hypothesis (23) holds in Kurtz's theorem. The approximating deterministic differential equation is

$$
\left(\begin{array}{l}
\dot{x} \\
\dot{y}
\end{array}\right)=\sum_{\left(k_{1}, k_{2}\right)}\left(\begin{array}{l}
k_{1} \\
k_{2}
\end{array}\right) \cdot f\left((x, y),\left(k_{1}, k_{2}\right)\right)=\left(\begin{array}{c}
-\frac{\lambda x y}{x+(y-1)}-\mu x+1 \\
+\frac{\lambda x y}{x+(y-1)}-(\mu+k) y
\end{array}\right) .
$$

By the theorem, if $\left(\mathscr{X}_{U}(t), \mathscr{Y}_{U}(t)\right)$ is a solution of (48) for $U$ large enough, then $(1 / U)\left(\mathscr{X}_{U}(t), \mathscr{H}_{U}(t)\right)$ converges to the globally stable equilibrium of (68):

$$
\frac{1}{\lambda-k}\left(1, \frac{1}{R_{0}}-1\right)
$$

In particular, $\mathscr{F}_{U}(t)$ converges weakly to

$$
\frac{U}{\lambda-k}\left(\frac{1}{R_{0}}-1\right)
$$

and

$$
\frac{\mathscr{X}_{U}(t)}{\mathscr{X}_{U}(t)+\mathscr{Y}_{U}(t)} \rightarrow \frac{1}{R_{0}}
$$

Notice that we could have used $x y /(x+y)$ instead of $x y /[x+(y-1)]$ in (68) because Kurtz's theorem gives an asymptotic result as $U \rightarrow \infty$, for which $x y /[x+(y-1)] \rightarrow x y /(x+y)$. 
5. COMPARISON OF DETERMINISTIC AND STOCHASTIC MODELS

Initial Time Course of Epidemic. From Equations (43) and (53), if the epidemic starts with a few infectives in a large population, then

$$
\frac{X}{X+Y} \approx \frac{x}{x+y-1} \approx 1 .
$$

In that case, Equations (43) and (53) become the same linear differential equation,

$$
\frac{d y}{d t}=(k+\mu)\left(R_{0}-1\right) y,
$$

with initial growth rate $(k+\mu)\left(R_{0}-1\right)$ [6].

Invariant Subspace of Equilibrium Solutions. From Equations (47) and (59), we see that the equilibrium solutions for the deterministic system (40), (41) and the stochastic means system (51), (53) fall on the same invariant subspace. From Equation (67), the equilibrium stochastic mean conditioned on nonextinction falls quite near this subspace. In fact, by the argument in Section III.B.4, for large $n$, it falls very close to the deterministic endemic equilibrium. Our simulations bear this out. Figure 13 indicates this subspace.

Comparison of $m^{* e}$ and $Y^{\mathrm{cq}}$. We can use the dynamics (64), (65) of the means conditioned on nonadsorption, $\left(m_{\mathscr{X}}^{*}, m_{\mathscr{Y}}^{*}\right)$, to shed some light on the comparison between the endemic equilibria of the deterministic (40), (41) and stochastic (48) models. We assume throughout this

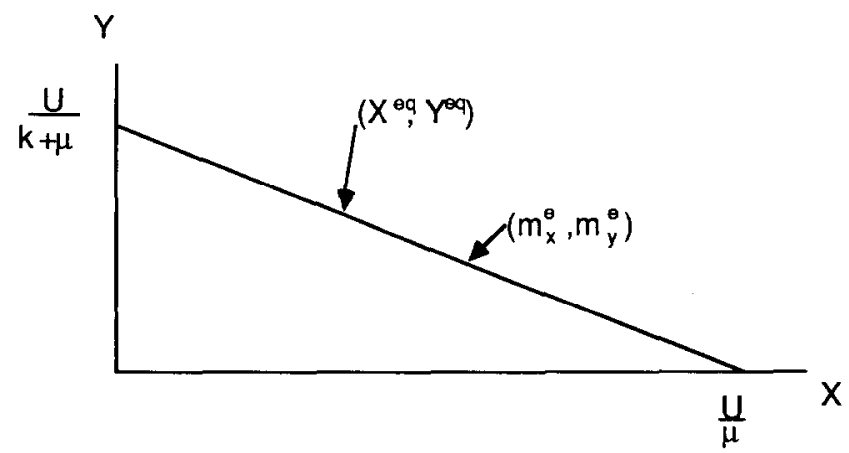

FIG. 13. Invariant subspace on which the deterministic and stochastic mean equilibria fall. 
discussion that $P_{1}^{*}=\sum_{x} q_{x 1}$ and $\sum_{x} x q_{x 1}$ go to zero for large enough $N$; see Equation (66) and the ensuing discussion.

Comparing (54) and (55), we note that

$$
\xi(t) m_{\mathscr{Y}}^{*}=\eta(t) m_{\mathscr{X}}^{*}=\sum_{x=0} \sum_{y=1} \frac{x y}{x+y-1} p_{x y}
$$

By (64) and (65), at the quasi-stationary solution,

$$
R_{0} \eta^{e} m_{\mathscr{X}}^{* e} \approx m_{\mathscr{Y}}^{* e}
$$

and

$$
U \approx\left[(k+\mu) R_{0} \eta^{e}+\mu\right] m_{\mathscr{x}}^{* e}
$$

Therefore,

$$
m^{* e} \approx \frac{U}{(k+\mu)+\mu / R_{0} \eta^{e}} .
$$

Compare (70) with the endemic equilibrium (45) of the deterministic system:

$$
Y^{\mathrm{eq}}=\frac{U}{(k+\mu)+\mu /\left(R_{0}-1\right)} .
$$

If $\mu=0$, then (70) and (71) yield

$$
Y^{\mathrm{eq}}=U / k \approx m^{* e} .
$$

Expressions (70) and (71) are also close if, in the denominators of (70) and (71), $(k+\mu)$ is much bigger than $\mu / R_{0} \eta^{e}$ and $\mu /\left(R_{0}-1\right)$.

On the other hand, if $\mu$ is large enough and $R_{0}>1$ small enough that the right-hand terms in the denominators of (70) and (71) dominate the left-hand term, $(k+\mu)$, then $m^{* e}$ and $Y^{\text {eq }}$ may differ some, even for rcasonably large $n$. Figure 24 (sec Section IV) presents an example of this phenomenon.

\section{THE SI MODEL: SIMULATIONS}

The simulations of the stochastic model were carried out on a SUN SPARCstation with use of double-precision arithmetic. The $p_{x y}$ were generated directly by integrating their system of simultaneous differential equations (48). Although $x$ and $y$ are defined on $[0, \infty)$, the $p_{x y}$ 's become negligible for $x$ and $y$ much larger than $n$, so for each 
simulation $p_{x y}$ was set to zero for $x$ and $y$ above certain bounds. At each time point a check sum of the $p_{x y}$, namely $\Sigma_{x} \Sigma_{y} p_{x y}$, was calculated. The check sums on all simulations were always equal to 1 , to within six decimal places.

We present no simulations for $R_{0} \leqslant 1$; the results are simple and adequately presented in [6] and in the text. Simulations for $R_{0}>1$ were run for $k=0.06, \mu=0.02$ for $n=10,30,100,300$. The recruitment rate $U$ was set to give the equilibrium number of susceptibles in the absence of infection, $U=n \mu . R_{0}$ was varied by changing the effective contact number $\lambda$. For each value of $n$, runs were obtained for $R_{0}=1.1,2,8$ and $a=1$ and 4 . For each simulation we calculated the marginal probabilities for $x$ and $y$ at a series of points in time:

$$
p_{. y}(t)=\sum_{x=0} p_{x y}(t), \quad p_{x .}(t)=\sum_{y=0} p_{x y}(t) .
$$

Note that $P_{\mathrm{ex}}=p_{0}$. In addition, the means $m_{\mathscr{P}}, m_{\mathscr{Y}}, m_{\mathscr{P}}^{*}, m_{\mathscr{Y}}^{*}$ and the variances and covariances were calculated.

In addition to the main runs, an example was run with $\mu=0.09$ and $k=0.01$ to show that $Y^{\text {eq }}$ and $m_{y}^{* e}$ can be separated.

\section{A. The Marginal Probabilities}

Figures 14 and 15 show the change with time in the marginal probabilities for $R_{0}=2, a=1$, and $n=10$ and 100 . The results for $R_{0}=8$ do not differ qualitatively and are not shown. The major findings can be seen by comparing the results for $n=100$ with those for $n=10$. Initially all of the probability mass is on $(x, y)=(n, 1)$. For $n=100$ (Figure 15), the probability mass distribution soon splits into two distributions. One has mass concentrated on $y=0$ and distributed in $x$ about mean $U / \mu$; the other has mass distributed around the means $m_{\mathscr{Z}}^{*}$ and $m_{\mathscr{Y}}^{*}$ conditioned on nonextinction. For $n=10$ (Figure 14), the picture is rather different. The probability mass on $y$ starts to split, but then it gradually falls back onto $y=0$ while the $x$ marginal becomes distributed around $m_{\mathscr{x}}=U / \mu$; here we see the eventual complete extinction of infection that is expected for models with an absorbing state. This is seen in our simulations only for $n=10$ and $n=30$ (not shown); we would have to run the simulations out to very long times indeed to pick it up for $n \geqslant 100$.

The corresponding plots for the marginal probabilities when four infecteds are introduced, $a=4$, are similar to Figures 14 and 15 except that less probability mass appears at $y=0$ in the early rise to a quasi-stationary level. For comparison we show only the plots for $n=100$ in Figure 16. 


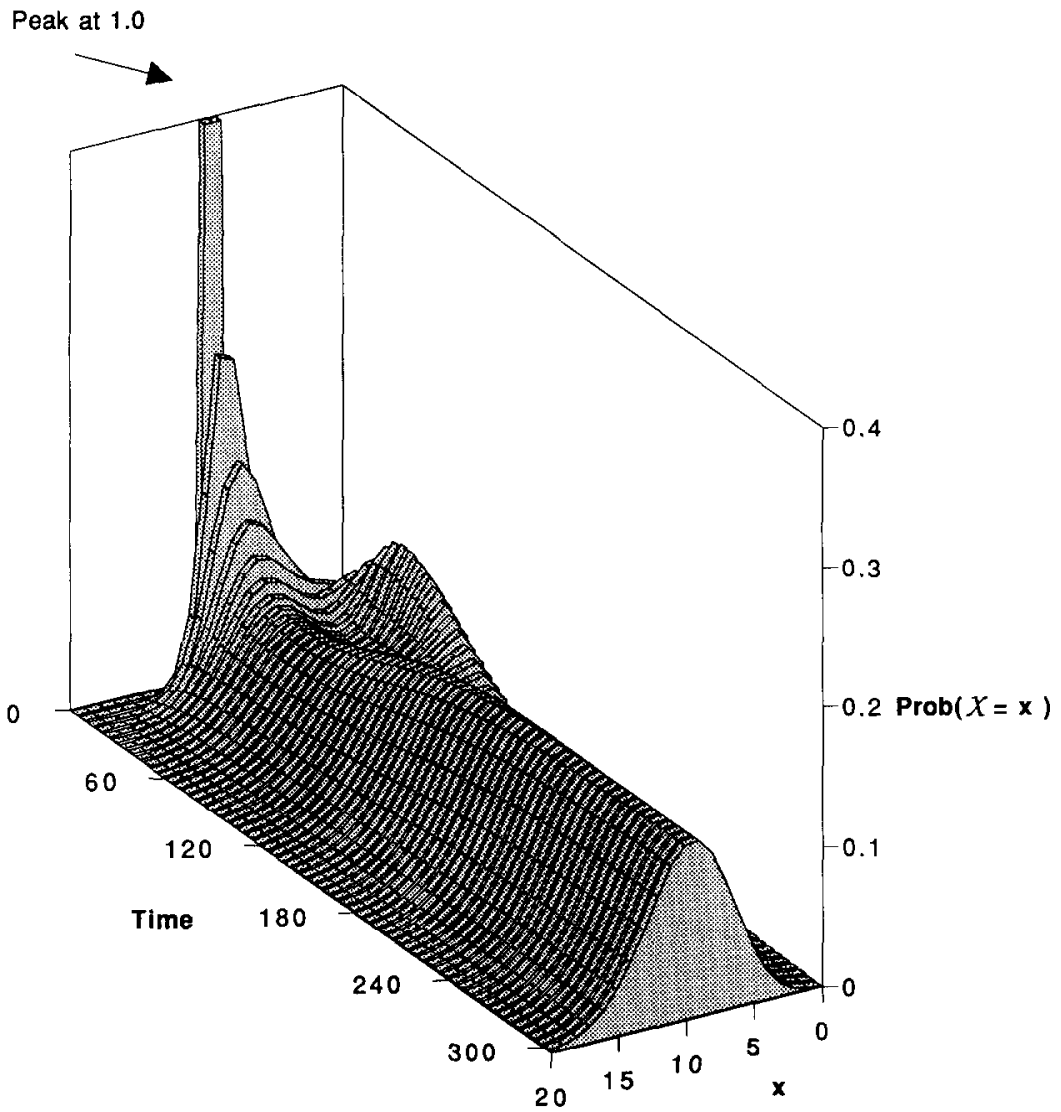

(a)

FIG. 14. Plots of marginal probabilities (a) in $x$ and (b) in $y$ for $R_{0}=2, n=10$, and $a=1$.

\section{B. The Probability of Extinction}

The probabilities of extinction, $P_{\text {ext }}(t)=p_{.0}(t)$, are plotted for $n=$ $10,30,100,300$ and with $a=1$ in Figure 17 and $a=4$ in Figure 18. There are a number of interesting features to these results.

$P_{e x t} \approx\left(1 / R_{0}\right)^{a}$ at the Quasi-Stationary Level. $P_{\mathrm{cxt}}$ rises to an early plateau that is near $\left(1 / R_{0}\right)^{a}$. Why? Early in the process, for $y$ small, and the more so the larger $x$ is, the generation of infectives is approximately a linear birth-and-death process, with birth rate $\lambda$ and death rate $k+\mu$ for each of the introduced infectives. Such a process has a probability of extinction of $[(k+\mu) / \lambda]^{a}=\left(1 / R_{0}\right)^{a}$. However, as $y$ 


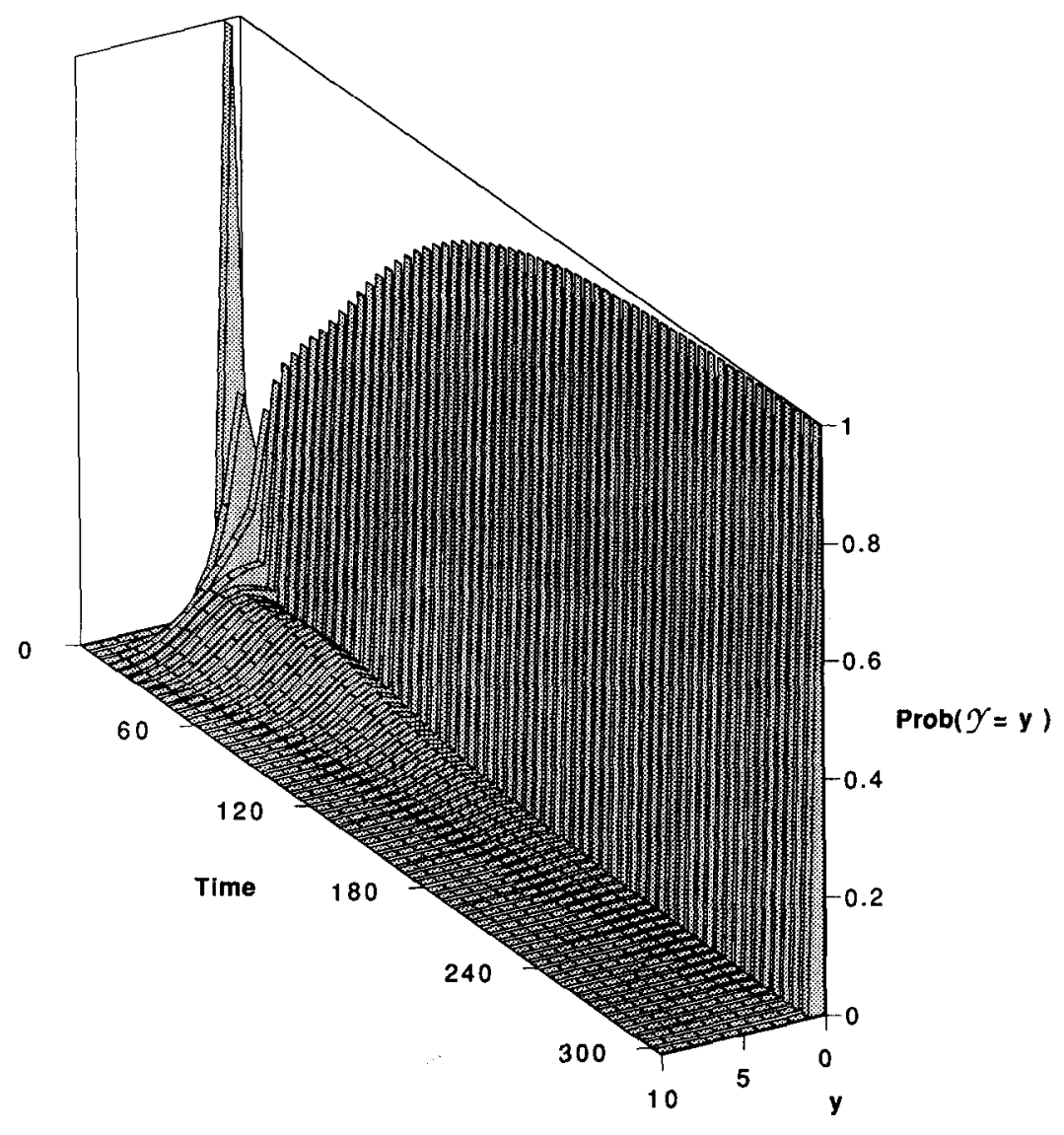

(b)

FIG. 14. (Continued).

increases, there is a progressive deviation from the linear birth-anddeath process. But, as is seen in the plots of the marginals, for $n$ even moderate in size, the probability mass distribution splits and the system comes to a quasi-stationary state. $P_{\text {ext }}$ is generated early while the process is still mostly a linear birth-and-death process and then remains at that quasi-stationary level after the split in the mass distribution. That is not true for $n=10$, for which $P_{\text {ext }}$ pauses momentarily near the quasi-stationary level and then continues to rise toward $P_{\mathrm{ext}}=1$. For $n=30$ the same occurs, but the system stays near the quasi-stationary level somewhat longer.

In the Long Term, $P_{\mathrm{cxt}} \rightarrow 1$. As just noted, $P_{\mathrm{cxt}}$ eventually goes to 1.0, 


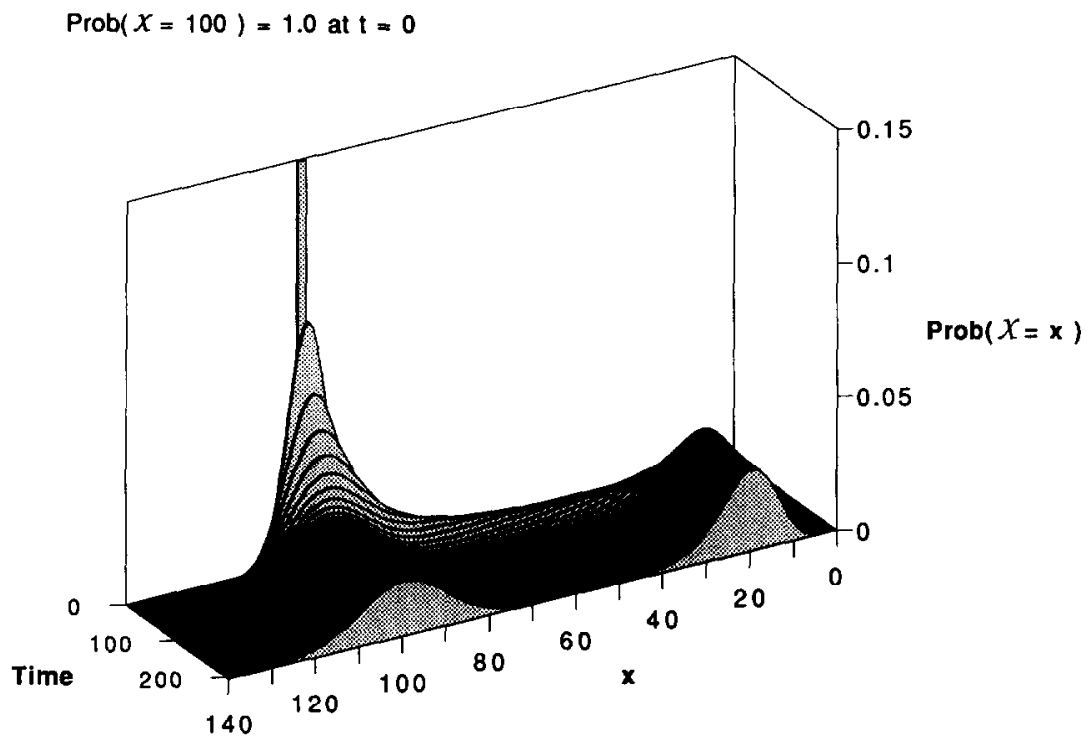

(a)

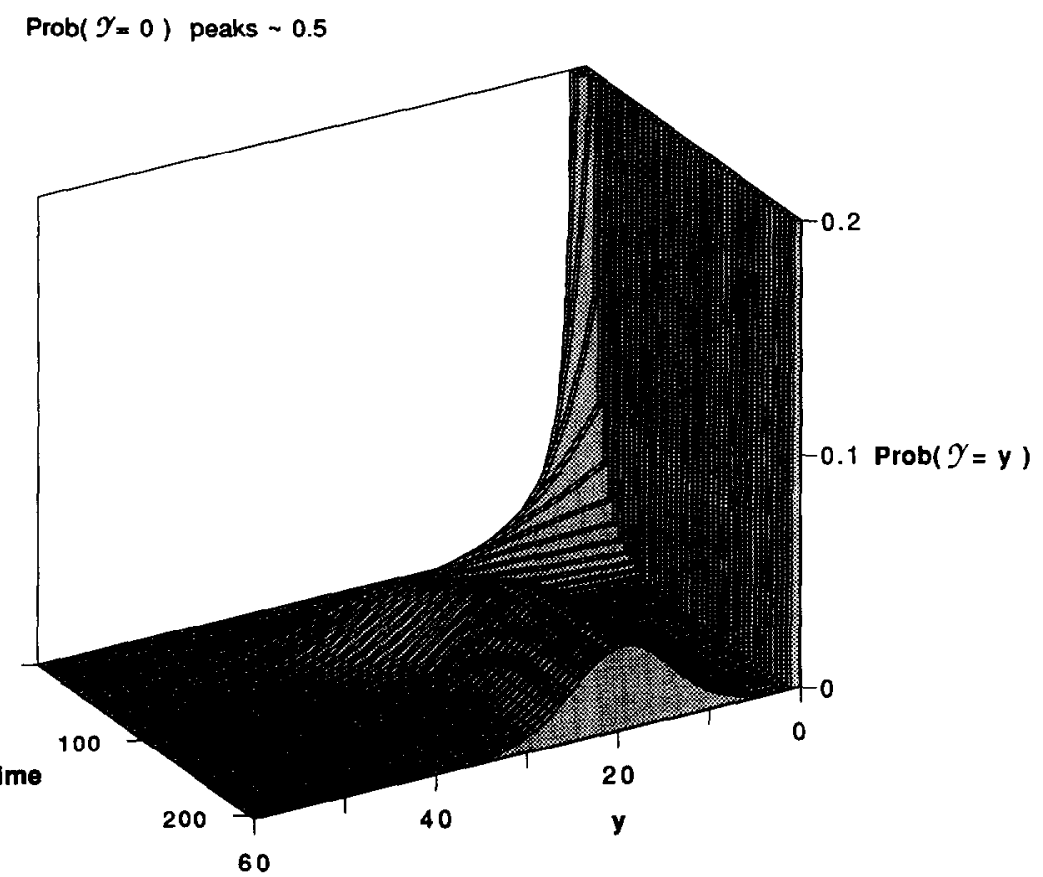

(b)

FIG. 15. Plots of marginal probabilities (a) in $x$ and (b) in $y$ for $R_{0}=2, n=100$, and $a=1$. 


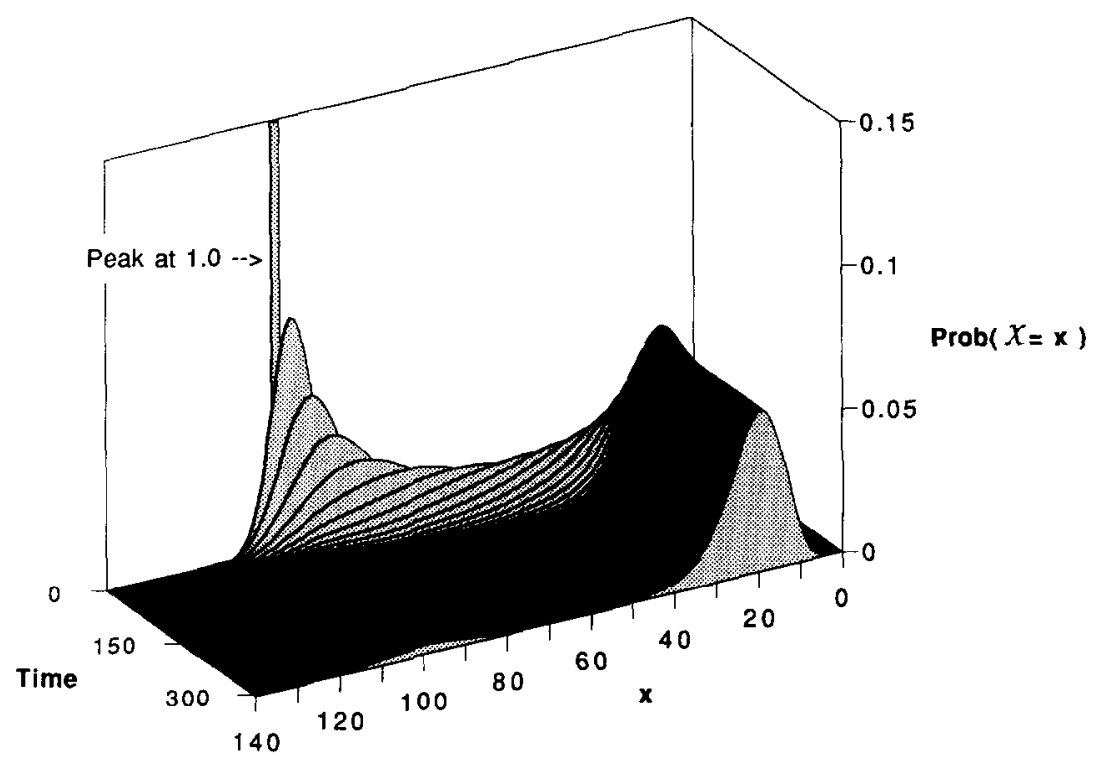

(a)

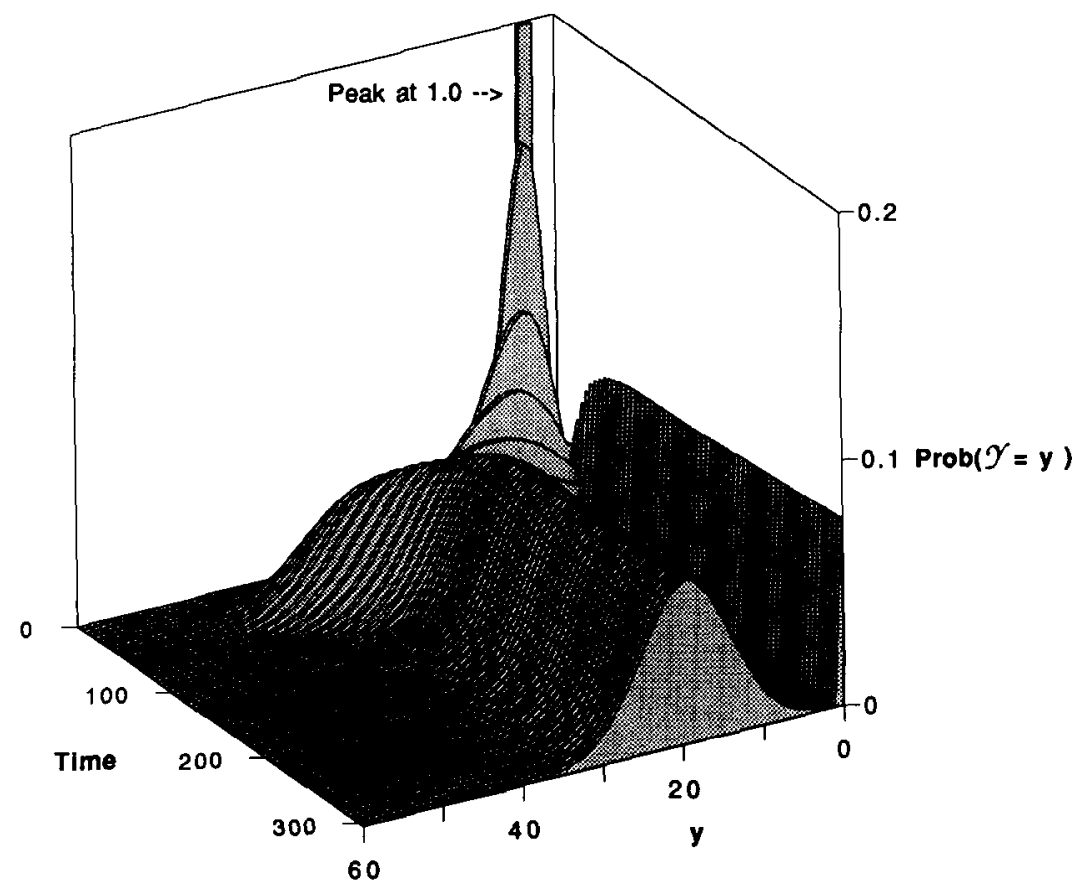

(b)

FIG. 16. Plot of marginal probabilities (a) in $x$ and (b) in $y$ for $R_{0}=2, n=100$, and $a=4$. 


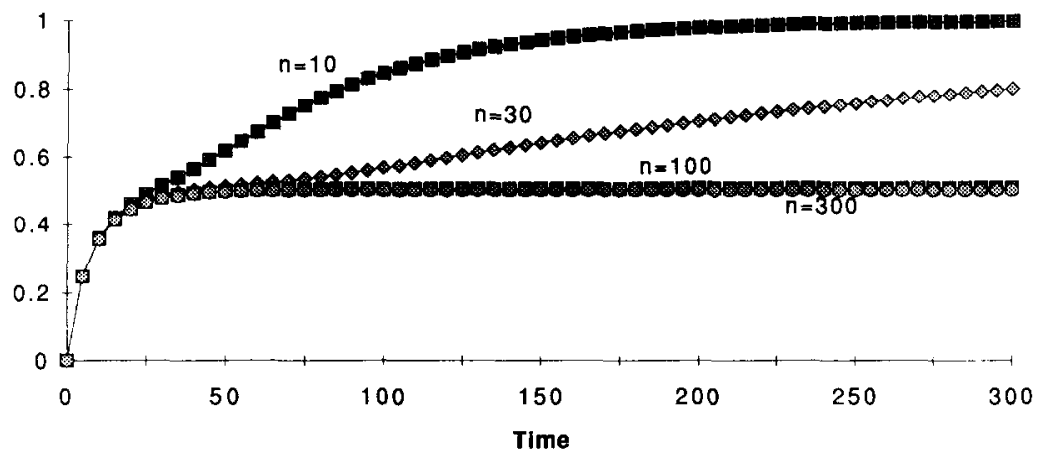

FIG. 17. Probability of extinction of the disease for $R_{0}=2$ and $a=1$ for $n=$ $10,30,100,300$.

but for $n$ even as small as 100 that takes so long we don't see it in our simulations. That is well illustrated in our results for $n=10$ and $n=30$. This is the same phenomenon as was seen in the SIS models, where the estimates of Oppenheim et al. [18], Norden [17], and Kryscio and Lefèvre [11] show that the quasi-stationary state is extremely long-lived for relatively small values of $N$.

Remark. The take-off toward $P_{\mathrm{ext}}=1$ was seen in our previous simulations [6] for $n=10$ and $n=30$ but was misinterpreted. In our previous studies the check sums began to deviate above 1.01 near the time when $P_{\mathrm{exl}}$ began to move up off the quasi-stationary level, so take-off was thought to be due to error accumulation. A careful check of our program brought to light a small error in programming that had

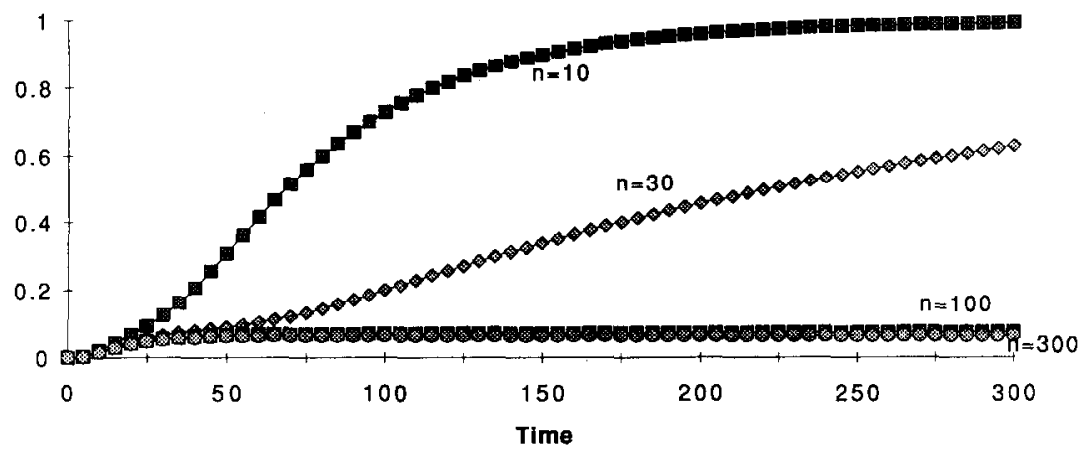

Fig. 18. Probability of extinction of the disease for $R_{0}=2$ and $a=4$ for $n=$ 10,30, 100,300. 
its major effect on the check sums for $n$ small. After correcting the error, all check sums remained at 1.0 to within six decimal places, but the take-off of $P_{\text {ext }}$ still occurred for $n=10$ and $n=30$.

C. The Means and the Quasi-Stationary State

Figure 19 plots $X, m_{\mathscr{X}}, m_{\mathscr{X}}^{*}$ and $Y, m_{\mathscr{Y}}, m_{\mathscr{Y}}^{*}$ for $n=10, a=1$, and $R_{0}=2.0$. Figure 20 gives the same plots for $n=100$. Note the long-lived near-stationary state for $n=100$ where $m_{Z^{\prime}}$ and $m_{Y}$ are essentially constant and $m_{\mathscr{Y}}^{*}$ and $m_{\mathscr{x}}^{*}$ are very close to $Y^{\mathrm{eq}}$ and $X^{\mathrm{eq}}$, respectively. However, for $n=10$, the near-stationary state is too short-lived to be of any consequence; $m_{y}$ falls steadily toward zero, so $m_{\xi}^{*}$ is irrelevant.

\section{$X$ means}

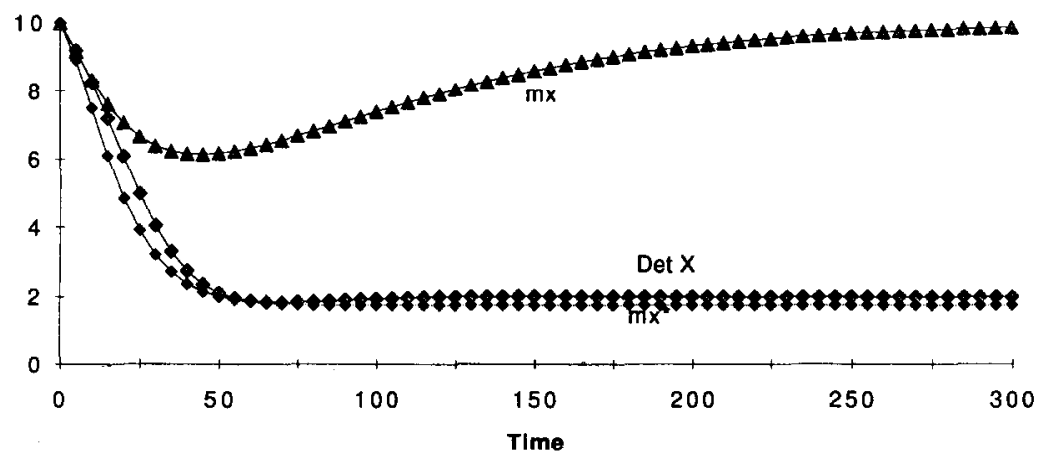

$\mathcal{Y}$ means

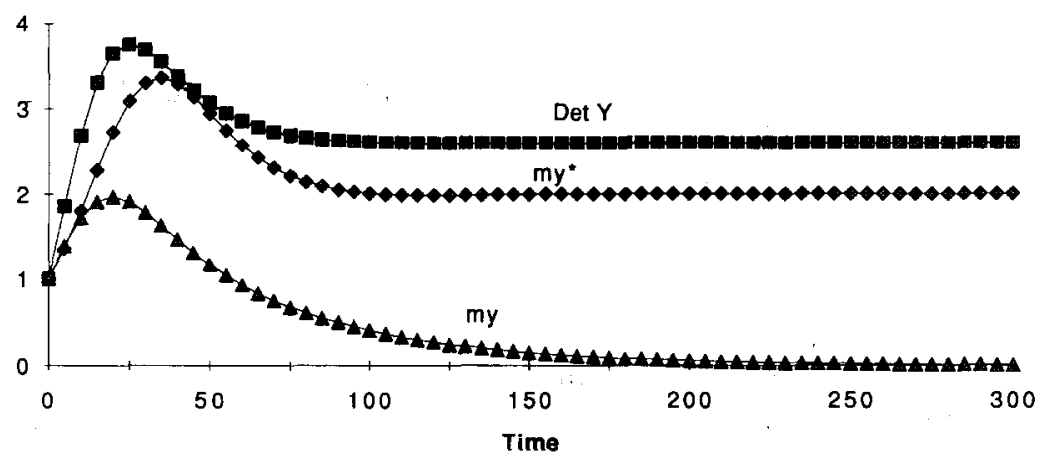

FIG. 19. Plots of $X, m_{\mathscr{x}}, m_{\mathscr{Z}^{*}}^{*}$ and $Y, m_{Z^{\prime}}, m_{Z^{\prime}}^{*}$ for $R_{0}=2, a=1$, and $n=10$. 


\section{$x$ means}

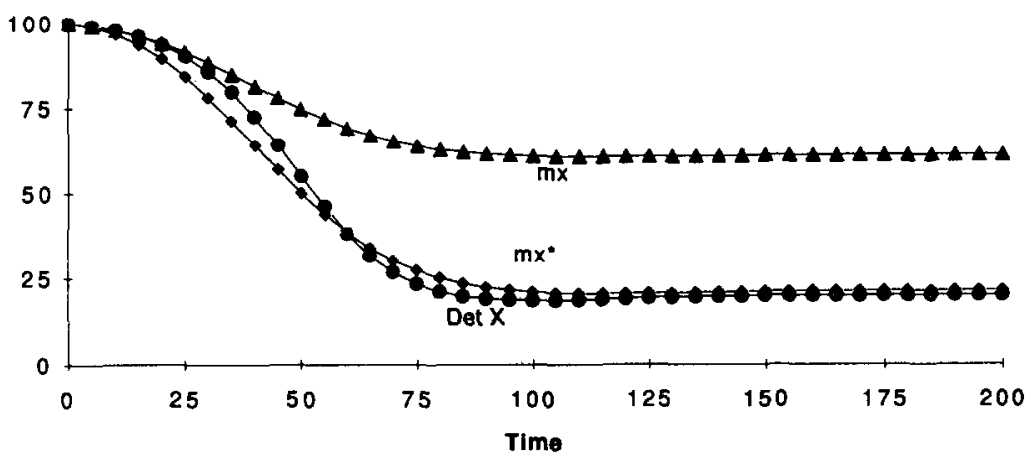

$\gamma$ means

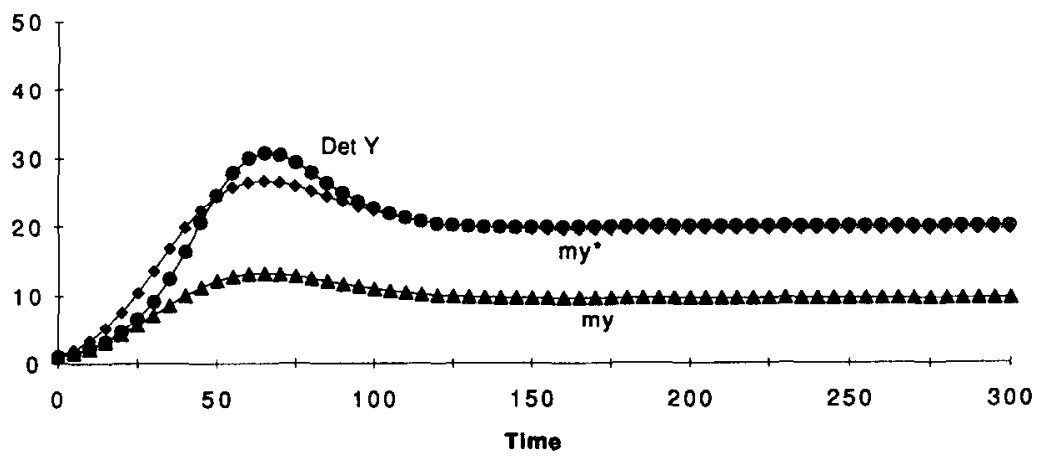

FIG. 20. Plots of $X, m_{\mathscr{P}}, m_{\mathscr{Z}}^{*}$ and $Y, m_{\mathscr{Y}}, m_{\mathscr{Y}}^{*}$ for $R_{0}=2, a=1$, and $n=100$.

Similar results were obtained for $a=4$, but, as expected, the larger the number of infecteds introduced, the lower the quasi-stationary level of $P_{\text {ext }}$, so $m_{y}$ and $m_{y}^{*}$ are closer in value. For comparison we show the results for $n=10$ and $n=100$ for $a=4$ in Figures 21 and 22, respectively.

In the simulations on the SIS model, $m_{y}$ rose monotonically to an obvious maximum for $n$ very small or to a quasi-stationary level for $n$ large. Numerically there was only one root to $R_{0} \xi(t)-1=0$. Figures such as Figure 20 raise a new possibility for the more complex SI model. There, $m_{y}$ rises to a relative maximum and then falls to an apparent quasi-stationary level. Does $R_{0} \xi(t)-1=0$ have more than one root? 


\section{$x$ means}

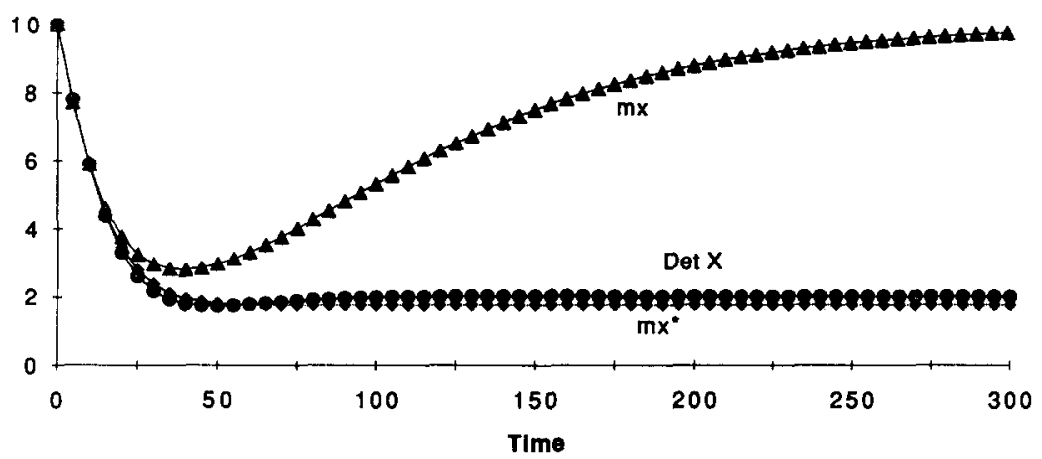

$Y$ means

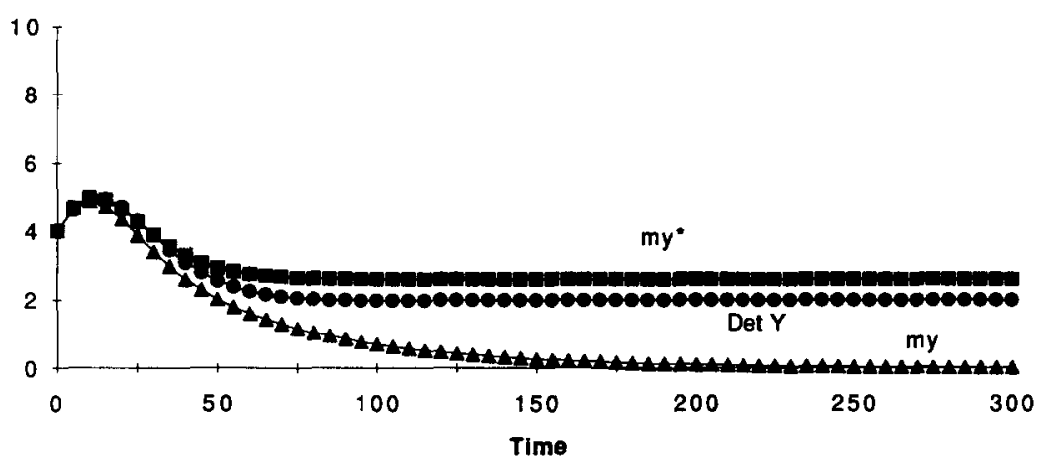

FIG. 21. Plots of $X, m_{\mathscr{X}}, m_{\mathscr{Z}}^{*}$ and $Y, m_{\mathscr{Y}}, m_{\mathscr{Y}}^{*}$ for $R_{0}=2, a=4$, and $n=10$.

The answer is sometimes yes. Figure 23 plots $\xi(t), \eta(t)$, and $R_{0} \xi(t)-1$ for $R_{0}=2$ and $N=100$. It shows an obvious root at about the peak in $m_{y}$. Numerically, there are two more roots, one near 175 time units and another near 225 time units. From 350 time units to the end of the simulation, which was at 500 time units, $R_{0} \xi(t)-1$ was constant at a value of -0.00043 .

In the results shown so far, $m_{y}^{*}$ was always very close to the deterministic value $Y$ for large $t$ and $n=100$, more so for $n=300$ (not shown). Hence we sought parametric combinations that would separate the two. Figure 24 shows results for $k=0.01, \mu=0.09, R_{0}=1.45$ in which $m_{y}^{* e}$ is clearly different from $Y^{\mathrm{eq}}$. 


\section{$X$ means}

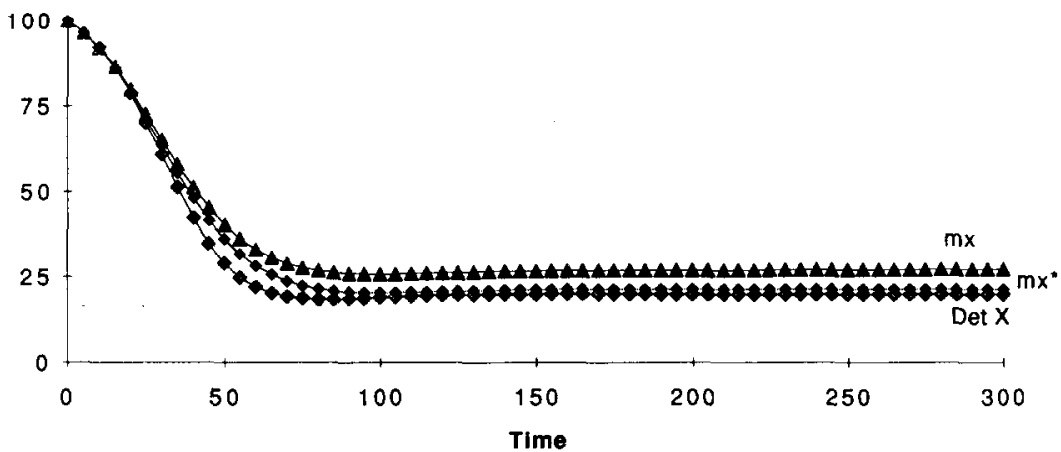

$Y_{\text {means }}$

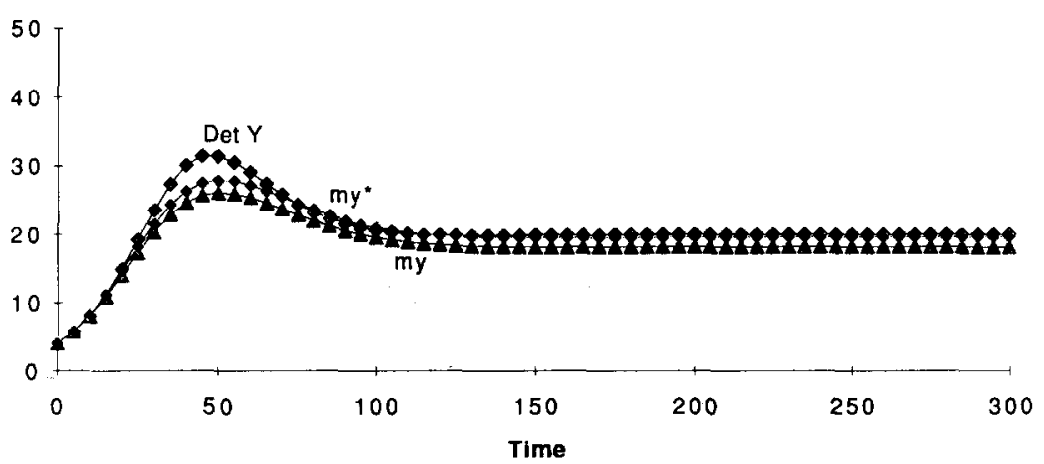

FIG. 22. Plots of $X, m_{\mathscr{Y}}, m_{Y^{\prime}}^{*}$ and $Y, m_{\mathscr{Y}}, m_{\mathscr{Y}}^{*}$ for $R_{0}=2, a=4$, and $n=100$.

D. Variances and Covariances

For every simulation, the variances of $\mathscr{X}$ and $\mathscr{Y}$ and their covariance were calculated for the distributions conditioned on nonextinction, that is, around the means $m_{\mathscr{X}}^{*}$ and $m_{\mathscr{Y}}^{*}$. All show much the same pattern-high values while the variables are changing rapidly and then relatively small variances and very small covariance for the quasistationary state. Figure 25 gives two examples.

\section{DISCUSSION AND CONCLUSIONS}

For the closed SIS (constant population size) model we have reviewed known results on the solution of the Kolmogorov equations, on 


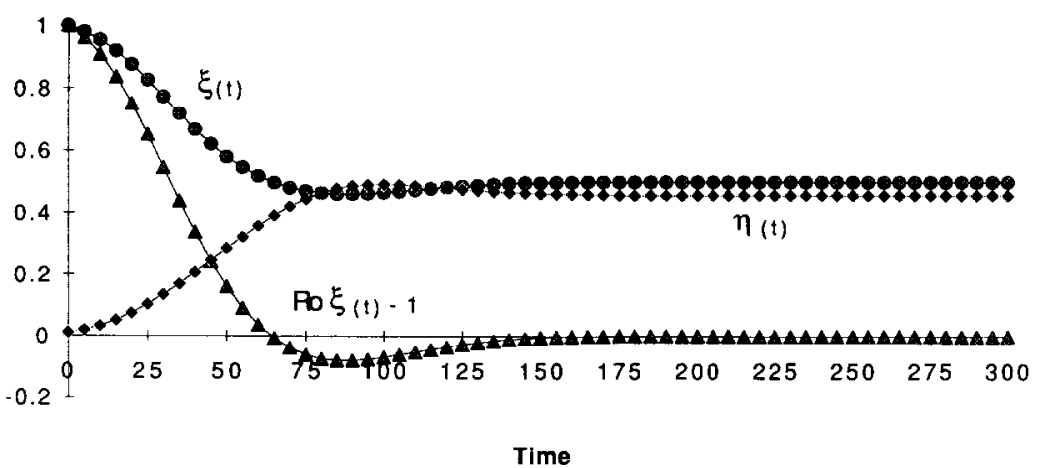

FIG. 23. Plot of $\eta(t), \xi(t)$, and $R_{0} \xi(t)-1$ for $R_{0}=2$ and $n=100$.

the quasi-stationary solutions obtained by conditioning on nonabsorption, on the approximation to the quasi-stationary solutions obtained by modifying the model to prevent absorption, and on the asymptotic results obtained with the use of Kurtz's theorems. To that, we have added an analysis of the dynamics of the mean number of infecteds, $m_{y}$, and the mean number conditioned on nonabsorption, $m_{\mathscr{Y}}^{*}$, coupled with simulation studies of some special cases. For $R_{0}>N /(N-1)$, we show that for $N$ small, $m_{y}$ has a relative maximum at the root of $R_{0} \xi(t)-1=0$ and then falls to zero. For such cases one can calculate a quasi-stationary solution $m_{\mathscr{Y}}^{*}$, but it is of little interest because there is no long, almost stationary plateau in $m_{\mathscr{y}}$ that corresponds to the endemic equilibrium of the deterministic model. However, for $N$ large, $m_{y}$ has a long nearly stationary plateau in which the relative maximum lies at the root of $R_{0} \xi(t)-1=0$. In this case, the quasi-stationary solution $m_{y}^{*}$ has meaning. Even for relatively small $N, m_{y}^{*}$ is close to the endemic solution of the deterministic model. By Kurtz's theorem, it converges asymptotically to the endemic plateau of the deterministic model as $N$ grows.

To the extent feasible, our goal is to carry out a similar program for the more complex SI model that has constant input to the susceptible class, background deaths for susceptibles and infecteds, and deaths due to the disease, so that the population is no longer constant. We show that Kurtz's theorems give asymptotic results as the parameter $U=n \mu$ goes to $\propto$. A start is made on studying the infinite-dimensional Kolmogorov systems of equations for this system. Much remains to be done with the approximations of the Kolmogorov equations analogous to systems (17) and (20) for the closed SIS model.

The analysis of the differential equations for the means, $m_{\mathscr{X}}, m_{\mathscr{Y}}$, 


\section{$X$ means}

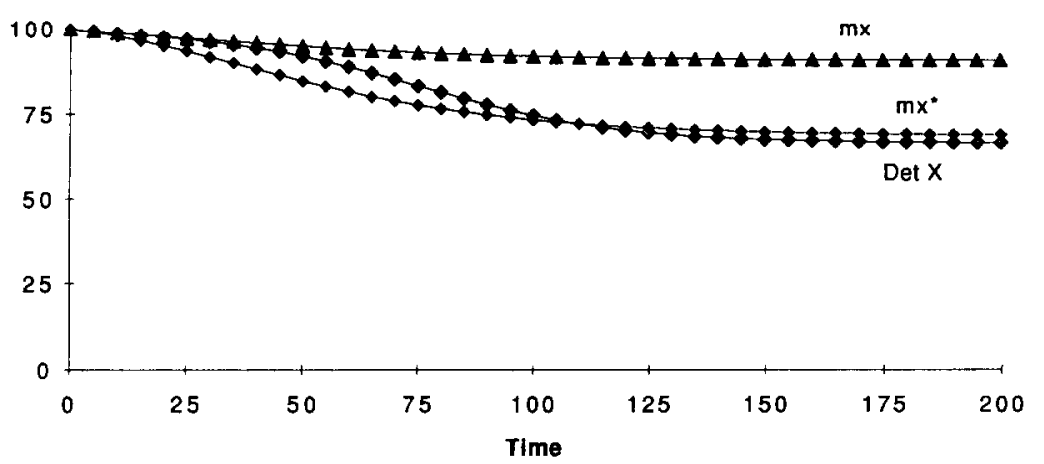

$y$ means

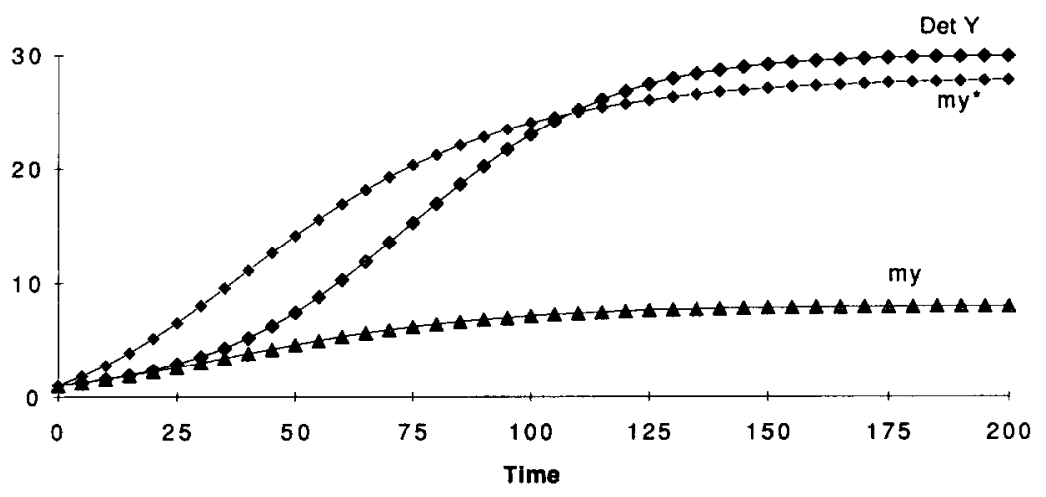

FIG. 24. Plots of means for $R_{0}=1.45, k=0.01$, and $\mu=0.09$ for $n=100$.

$m_{\mathscr{x}}^{*}$, and $m_{\mathscr{w}}^{*}$, coupled with simulation results, have given us a clearer picture of the dynamics of the stochastic model of this system.

(1) Early in the process the probability of extinction rises to a value that is close to $\left(1 / R_{0}\right)^{a}$, where $a$ is the initial number of infecteds introduced into the population. We were at first surprised by this behavior because the system is nonlinear. However, the simulations indicated that the probability mass distributions split fairly early into a single mass on $y=0$ separated from an apparently symmetric probability distribution for $y>0$. This early split occurred while the system was 


\section{Varlance*}

$$
\text { Ro }=2.0, n=10
$$

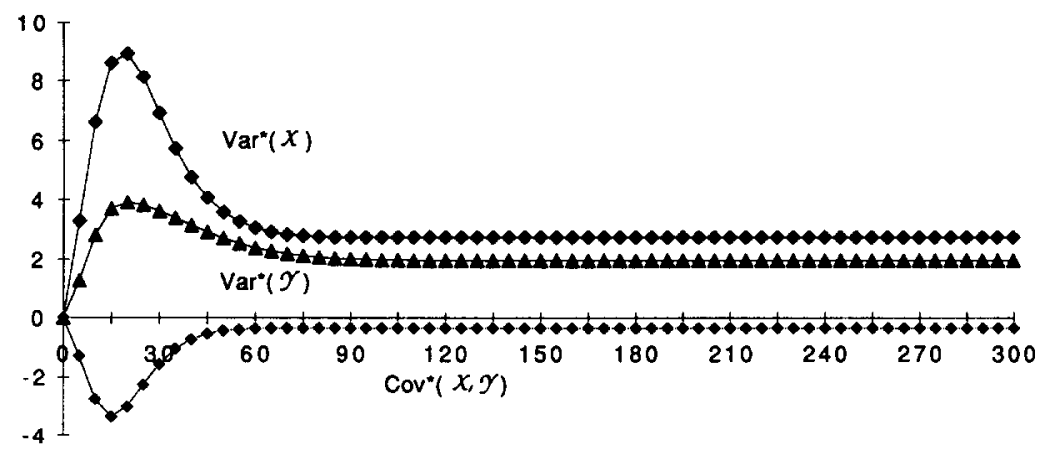

$R_{0}=2.0, n=100$

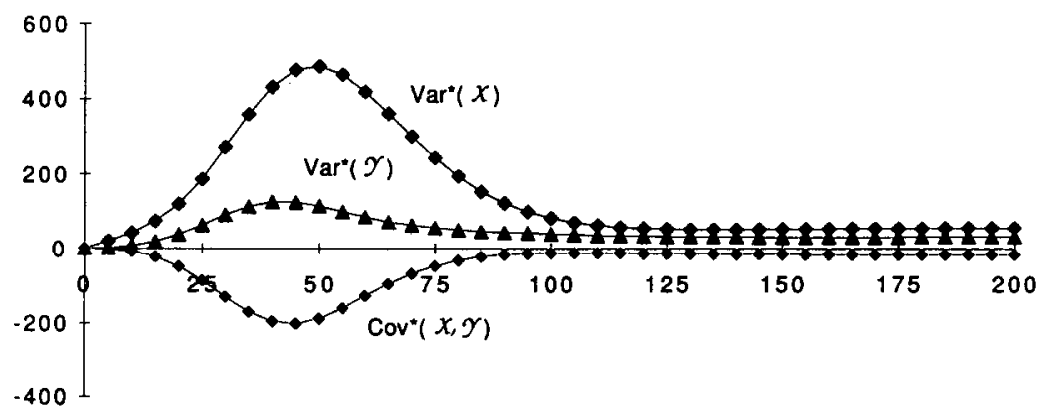

Time

Fig. 25. Plots of the variances and covariances of $\mathscr{Z}$ and $\mathscr{Y}$ for $R_{0}=2, a=1$ for $n=10$ and $n=100$. 
still close to linear in behavior and we know that the probability of extinction for a linear birth-and-death process is exactly $\left(1 / R_{0}\right)^{a}$.

(2) We argue that the solutions for $m_{Y}^{*}$ and $Y$ at equilibrium must be quite close to each other for a large range of parameter values for $n$ fairly small. By Kurtz's theorems, they approach each other asymptotically as $n \rightarrow \infty$ for all parameter values.

(3) For $n$ small, $m_{y /}$ rises to a maximum and then, as expected, falls steadily toward zero. Nonetheless the quasi-stationary solution shows a plateau somewhere near but not close to the endemic solution of the deterministic model; both the deterministic solution and the quasistationary solution are irrelevant to the dynamics in these circumstances. For $n$ large, $m_{y}$ rises to long-lived, relatively flat plateau; then the quasi-stationary state is close to the endemic solution of the deterministic model. In that case, the deterministic solution may be a good approximation to the stochastic dynamics when the disease takes off, that is, the quasi-stationary solution.

We thank the guest editors and the anonymous referees for insightful critiques and suggestions for revision. This work was supported in part by grant RO1 AI29876 from NLAID, DHEW, and by RR02176 from the National Center for Research Resources, DHEW.

\section{REFERENCES}

1 N. T. J. Bailey, The Mathematical Theory of Infectious Diseases, 2nd ed., Charles Griffin, London, 1975.

2 F. C. Ball and P. D. O'Neill, A modification of the general stochastic epidemic motivated by AIDS modeling, Adv. Appl. Probab. 25:39-62 (1993).

3 M. S. Bartlett, Deterministic and stochastic models for recurrent epidemics, Proc: 3rd Berkeley Symp. Muth. Stut. Prolub. 4:81-109 (1956).

4 O. Diekmann, J. A. P. Heesterbeek, and J. A. J. Metz, On the definition and the computation of the basic reproduction ratio $R_{0}$ in models for infectious diseases in heterogeneous populations, J. Math. Biol. 28:365-382 (1990).

5 J. Gani and P. Purdue, Matrix-geometric methods for the general stochastic epidemic, IMA J. Math. Appl. Med. Biol. 1:333-342 (1984).

6 J. A. Jacquez and P. O'Neill, Reproduction numbers and thresholds in stochastic epidemic models. I. Homogeneous populations, Math. Biosci. 107:161-186 (1991).

7 J. A. Jacquez and C. P. Simon, Qualitative theory of compartmental systems, SLAM Rev. 35:43-79 (1993).

8 J. A. Jacquez, C. P. Simon, J. S. Koopman, L. Sattenspiel, and T. Perry, Modeling and analyzing HIV transmission: the effect of contact patterns, Math. Biosci. 92:119-199 (1988).

9 J. A. Jacquez, C. P. Simon, and J. S. Koopman, The reproduction number in deterministic models of contagious diseases, Comments Theor. Biol. 2:159-209 (1991). 
10 W. O. Kermack and A. G. McKendrick, A contribution to the mathematical theory of epidemics, Proc. Roy. Soc. Lond. A115:700-721 (1927).

11 R. I. Kryscio and C. I efèvre, On the extinction of the S-I-S stochastic logistic epidemic, J. Appl. Probab. 27:685-694 (1989).

12 T. G. Kurtz, Solutions of ordinary differential equations as limits of pure jump Markov processes, J. Appl. Probab. 7:49-58 (1970).

13 T. G. Kurtz, Limit theorems for sequences of jump Markov processes approximating ordinary differential processes, J. Appl. Probab. 8:344-356 (1971).

14 C. Lefèvre and P. Picard, An epidemic model with fatal risk, Math. Biosci, this issue.

15 A. Martin-Löf, Diffusion approximation near the threshold in the stochastic SIS epidemic, presented at Luminy Conference on Stochastic Modeling for Infectious Diseases, November 1991.

16 I. Nåsell, The threshold of the stochastic SIS epidemic, presented at Luminy Conference on Stochastic Modeling for Infectious Diseases, November 1991.

17 R. H. Norden, On the distribution of the time to extinction in the stochastic logistic population model, Adv. Appl. Probab. 14:687-708 (1982).

18 I. Oppenheim, K. E. Shuler, and G. H. Weiss, Stochastic theory of nonlinear rate processes with multiple stationary states, Physica 88A 191-214 (1977).

19 P. Picard, Sur les modèles stochastiques logistiques en demographie, Ann. Inst. Henri Poincaré B 2:151-172 (1965).

20 C. J. Ridler-Rowe, On a stochastic model of an epidemic, J. Appl. Probab. 4:19-33 (1967).

21 C. P. Simon and J. A. Jacquez, Reproduction numbers and the stability of equilibria of SI models for heterogeneous populations, SIAM J. Appl. Math. 52:541-576 (1992).

22 D. R. Stirzaker, A perturbation method for the stochastic recurrent epidemic, $J$. Inst. Math. Appl. 15:135-160 (1975).

23 G. H. Weiss and M. Dishon, On the asymptotic behavior of the stochastic and deterministic models of an epidemic, Math. Biosci. 11:261-265 (1971).

24 P. Whittle, The outcome of a stochastic epidemic - a note on Bailey's paper. Biometrika 42:116-122 (1955).

25 T. Williams, An algebraic proof of the threshold theorem for the general stochastic epidemic, Adv. Appl. Probab. 3:223 (1971). 